\title{
Some empirical estimates of the $\mathrm{H}_{2}$ formation rate in photon-dominated regions
}

\author{
E. Habart ${ }^{1}$, F. Boulanger ${ }^{2}$, L. Verstraete ${ }^{2}$, C. M. Walmsley ${ }^{1}$, and G. Pineau des Forêts ${ }^{2}$ \\ 1 Osservatorio Astrofisico di Arcetri, INAF, Largo E. Fermi 5, 50125 Firenze, Italy \\ ${ }^{2}$ Institut d'Astrophysique Spatiale, Université Paris-Sud, 91405 Orsay Cedex, France
}

Received 20 December 2002 / Accepted 25 July 2003

\begin{abstract}
We combine recent ISO observations of the vibrational ground state lines of $\mathrm{H}_{2}$ towards Photon-Dominated Regions (PDRs) with observations of vibrationally excited states made with ground-based telescopes in order to constrain the formation rate of $\mathrm{H}_{2}$ on grain surfaces under the physical conditions in the layers responsible for $\mathrm{H}_{2}$ emission. We briefly review the data available for five nearby PDRs. We use steady state PDR models in order to examine the sensitivity of different $\mathrm{H}_{2}$ line ratios to the $\mathrm{H}_{2}$ formation rate $R_{\mathrm{f}}$. We show that the ratio of the $0-0 \mathrm{~S}(3)$ to the 1-0 S(1) line increases with $R_{\mathrm{f}}$ but that one requires independent estimates of the radiation field incident upon the PDR and the density in order to infer $R_{\mathrm{f}}$ from the $\mathrm{H}_{2}$ line data. We confirm earlier work by Habart et al. (2003) on the Oph W PDR which showed that an $\mathrm{H}_{2}$ formation rate higher than the standard value of $3 \times 10^{-17} \mathrm{~cm}^{3} \mathrm{~s}^{-1}$ inferred from UV observations of diffuse clouds is needed to explain the observed $\mathrm{H}_{2}$ excitation. From comparison of the ISO and ground-based data, we find that moderately excited PDRs such as Oph W, S140 and IC 63 require an $\mathrm{H}_{2}$ formation rate of about five times the standard value whereas the data for PDRs with a higher incident radiation field such as NGC 2023 and the Orion Bar can be explained with the standard value of $R_{\mathrm{f}}$. We compare also the $\mathrm{H}_{2} 1-0 \mathrm{~S}(1)$ line intensities with the emission in PAH features and find a rough scaling of the ratio of these quantities with the ratio of local density to radiation field. This suggests but does not prove that formation of $\mathrm{H}_{2}$ on PAHs is important in PDRs. We also consider some empirical models of the $\mathrm{H}_{2}$ formation process with the aim of explaining these results. Here we consider both formation on classical grains of size roughly $0.1 \mu \mathrm{m}$ and on very small $(\sim 10 \AA)$ grains by either direct recombination from the gas phase (Eley-Rideal mechanism) or recombination of physisorbed $\mathrm{H}$ atoms with atoms in a chemisorbed site. We conclude that indirect chemisorption where a physisorbed $\mathrm{H}$-atom scans the grain surface before recombining with a chemisorbed $\mathrm{H}$-atom is most promising in PDRs. Moreover small grains which dominate the total grain surface and spend most of their time at relatively low (below $30 \mathrm{~K}$ for $\chi \leq 3000$ ) temperatures may be the most promising surface for forming $\mathrm{H}_{2}$ in PDRs.
\end{abstract}

Key words. ISM: clouds - ISM: dust, extinction - atomic processes - molecular processes - radiative transfer

\section{Introduction}

The formation of molecular hydrogen is a key process affecting the thermal and density structure and the chemical evolution of the interstellar medium (see, for example, Combes \& Pineau des Forets 2000). Although there is a consensus that $\mathrm{H}_{2}$ forms on the surface of dust grains (Gould \& Salpeter 1963; Hollenbach \& Salpeter 1971; Jura 1975; Duley \& Williams 1984), the mechanism is not yet understood. This is partly due to our ignorance concerning interstellar grain composition, form, structure and physico-chemical state. It is also caused by our lack of understanding of surface reactions in the interstellar context.

Numerous theoretical and experimental studies have thus been dedicated to the study of the $\mathrm{H}_{2}$ formation process (Sandford \& Allamandola 1993; Duley 1996; Parneix \& Brechignac 1998; Pirronello et al. 1997, 1999; Takahashi et al. 1999; Katz et al. 1999; Williams et al. 2000; Sidis et al. 2000;

Send offprint requests to: E. Habart,

e-mail: habart@arcetri.astro.it
Biham et al. 2001; Joblin et al. 2001; Cazaux \& Tielens 2002, 2003). Another approach to this issue is to examine the $\mathrm{H}_{2}$ formation rate in different regions of the ISM in order to see how it depends upon the local physical parameters. One can for example study the correlation of $\mathrm{H}_{2}$-related quantities (abundance, rotational excitation, vibrational excitation) with the local dust properties. ISO observations of $\mathrm{H}_{2}$ lines and small carbonaceous grains emission in several PDRs, and new $\mathrm{H}_{2} \mathrm{UV}$ absorption observations by FUSE of diffuse clouds, open new perspectives on our understanding of the $\mathrm{H}_{2}$ formation process. In particular, the confrontation between observations and theoretical predictions provides strong constraints upon the $\mathrm{H}_{2}$ formation rate (Habart et al. 2003; Gry et al. 2002; Tumlinson et al. 2002).

In this paper, we consider PDRs and investigate the $\mathrm{H}_{2}$ formation process using observations of $\mathrm{H}_{2}$ emission obtained by ISO and ground-based telescopes. PDRs, where stellar radiation plays a dominant role in determining the chemical and thermal state of the gas (for a recent review see Hollenbach \& Tielens 1999), are privileged objects for the study of physical 
and chemical processes of the interstellar medium. In Sect. 2, we review the data available for five nearby PDRs and summarize their physical conditions. In Sect. 3, we estimate for each PDR of our sample the $\mathrm{H}_{2}$ formation rate using the $\mathrm{H}_{2}$ line intensity ratios as a diagnostic. Also, in order to probe the influence of PAHs on the $\mathrm{H}_{2}$ formation, we compare in Sect. 4 the intensities of emission measured in $\mathrm{H}_{2}$ fluorescent lines with those in the aromatic bands. Then, with the aim of explaining these results, we consider in Sect. 5 empirical models of the $\mathrm{H}_{2}$ formation mechansim by recombination of an $\mathrm{H}$ atom in a bound site with a second $\mathrm{H}$ atom which is either in the gas phase or on a neighbouring physisorbed site. In Sect. 6, we compare the observational constraints on the $\mathrm{H}_{2}$ formation rate with different model predictions. Our conclusions are summarized in Sect. 7.

\section{Sample of PDRs observed by ISO}

Recently, the Short Wavelength Spectrometer (SWS, Kessler et al. 1996) on board ISO has observed a series of pure $\mathrm{H}_{2}$ rotational lines towards a variety of nearby PDRs. In the ISO data base, we have selected five nearby PDRs which sample well the range of excitation conditions covered by the SWS observations. At the low excitation end, we have the Oph $\mathrm{W}$ and the S140 PDRs. Then, with radiation field higher by a factor of 3-10, we have the PDR IC 63 and the reflection nebula NGC 2023 (where we focus on the filament at $60^{\prime \prime}$ south of the central star). Finally, at the high excitation end, we have the Orion Bar at the position of the peak of the fluorescent $\mathrm{H}_{2}$ emission. These PDRs close to the Sun $(d \sim 100-500 \mathrm{pc})$ are ideal targets to discuss the formation of $\mathrm{H}_{2}$ in hot regions of the ISM.

In Table 1, we summarize the physical conditions prevailing in each region as determined from the literature. The thermal and chemical structure of the PDR depends on two parameters, namely, the intensity of the incident far-ultraviolet (FUV, $6<h v<13.6 \mathrm{eV}$ ) radiation field and the gas density. We adopt the radiation field of Draine (1978) and we characterize its intensity with a scaling factor $\chi(\chi=1$ corresponds to the standard FUV interstellar radiation field of $2.6 \times$ $10^{-3} \mathrm{erg} \mathrm{s}^{-1} \mathrm{~cm}^{-2}$ ). This factor is determined from the expected FUV luminosity of the exciting star and assuming that the distance of the exciting star to the PDR is equal to the distance projected onto the sky. This in principle is an upper limit and hence we also report in Table 1 estimations of $\chi$ based on observations of the fine structure lines of $\mathrm{C}^{+}$or $\mathrm{O}^{0}$. In general, we note that $\chi$ is uncertain by a factor of about 2 to 5 .

The proton gas density $n_{\mathrm{H}}$ has been derived from a variety of observational constraints: (1) for Oph W, $n_{\mathrm{H}} \sim 10^{4} \mathrm{~cm}^{-3}$ is estimated from the brightness profile of the aromatic dust emission (Habart et al. 2003), while observations in the $\mathrm{C}^{0}$ and CO lines suggest $n_{\mathrm{H}} \sim 6 \times 10^{4} \mathrm{~cm}^{-3}$ for the inner part of the PDR (Habart 2001); (2) for S140, using [C $\left.{ }^{+}\right] 158 \mu \mathrm{m}$ and $\mathrm{C}^{0}$ radio recombination line, Wyrowski et al. (1997b) derive a density about $n_{\mathrm{H}} \sim 5 \times 10^{4} \mathrm{~cm}^{-3}$ comparable to the gas density expected for pressure balance with the HII region $\sim 10^{4} \mathrm{~cm}^{-3}$ (Timmermann et al. 1996); (3) measurements of $\mathrm{CO}, \mathrm{HCO}^{+}, \mathrm{HCN}$ or CS line ratios from the PDR IC 63 suggest $n_{\mathrm{H}} \sim 5 \times 10^{4}-10^{5} \mathrm{~cm}^{-3}$ (Jansen et al. 1994, 1995);
(4) for the bright southern emission bar of NGC 2023, the inferred densities are in the range $\sim 10^{4}-10^{5} \mathrm{~cm}^{-3}$ using $\mathrm{H}_{2}$ fluorescent line emission (Black \& van Dishoeck 1987; Draine \& Bertoldi 1996; Field et al. 1998; Draine \& Bertoldi 2000) and $\sim 10^{5} \mathrm{~cm}^{-3}$ from $\left[\mathrm{C}^{+}\right] 158 \mu \mathrm{m}$ and $\mathrm{C}$ radio recombination line intensity (Wyrowski et al. 1997b, 2000); (5) finally, for the Orion Bar the gas density has been estimated to be about $\sim 5 \times 10^{4} \mathrm{~cm}^{-3}$ from the observed stratification of different tracers of PDRs (Tielens et al. 1993) and $\gtrsim 10^{5} \mathrm{~cm}^{-3}$ from observations of fine-structure line emission (Herrmann et al. 1997), as well as C radio recombination lines (Wyrowski et al. 1997a) and CN, CS observations (Simon et al. 1997). The gas density inferred from various atomic/molecular species shows a relatively large dispersion (typically from $10^{4}$ to $10^{5} \mathrm{~cm}^{-3}$ ). This dispersion could result from systematic density gradients from the $\mathrm{H}_{2}$ emitting layer to the molecular cold layer (see, for example, Walmsley et al. 2000; Habart et al. 2003). What is needed for our study is the density in the $\mathrm{H}_{2}$ emitting region while mainly the density tracers reflect the density of the cold gas in the cloud.

We report also in Table 1 the observed intensities for the $\mathrm{H}_{2} \quad 0-0 \mathrm{~S}(3)$ and 1-0 S(1) lines obtained respectively with ISO-SWS and from ground based observations. High spatial resolution observations $\left(\sim 1^{\prime \prime}\right)$ of the 1-0 S(1) line emission have been smoothed to the $\sim 20^{\prime \prime}$ beam of SWS. For measurements made with beams larger than the SWS observations, we have scaled the flux according to the beam ratio. For each region, we also give the excitation temperature of the $\mathrm{H}_{2}$ pure rotational levels with $J \leq 7$, the $\mathrm{H}_{2}$ column density inferred from $\mathrm{H}_{2}$ rotational lines intensity, and the 1-0 S(1)/2-1 S(1) line ratio. In the case of NGC 2023 and the Orion Bar, the 1-0 $\mathrm{S}(1) / 2-1 \mathrm{~S}(1)$ line ratio has been taken respectively at $18^{\prime \prime} \mathrm{S} 11^{\prime \prime} \mathrm{W}$ (Burton et al. 1998) and 50"N 30"E (Walmsley et al. 2000) of the SWS pointings. Finally, based on ISOCAM observations $^{1}$, we give the aromatic dust emission smoothed to the SWS beam. In the following, aromatic dust particles will be hereafter referred to as PAHs (Polycyclic Aromatic Hydrocarbons). This is a generic term which encompasses large aromatic molecules and tiny carbonaceous dust grains containing up to a few 1000 atoms and with radii of a few $\AA$ to a few tens of $\AA$.

\section{Estimates of the $\mathrm{H}_{2}$ formation rate}

In this section, we show how the $\mathrm{H}_{2}$ formation rate can be derived from the analysis of the $\mathrm{H}_{2}$ emission line using PDR models.

For several PDRs observed by ISO, the $\mathrm{H}_{2}$ line intensities and the gas temperature as probed by the populations of the low rotational levels of $\mathrm{H}_{2}$ were found to be higher than predicted by current models (Bertoldi 1997; Draine \& Bertoldi 1999; Thi et al. 1999; Habart et al. 2003; Li et al. 2002). The cause of this

1 From the brightness in the LW2 filter $(5-8.5 \mu \mathrm{m})$, which is dominated by the aromatic dust emission (Boulanger et al. 1998), we can estimate the aromatic dust emission using the following relationship : $I_{\mathrm{PAH}}(2-15 \mu \mathrm{m}) \simeq 2 \times v I_{v}(5-8.5 \mu \mathrm{m})$ based on ISOCAM-CVF spectrum (corrected from the dust continuum emission) taken in PDRs. 
Table 1. Sample of PDRs observed by ISO.

\begin{tabular}{llllll}
\hline \hline & Oph W & S140 & IC 63 & NGC 2023 & Orion Bar \\
\hline$\chi^{a}$ & 250 & $100-250$ & 650 & $500-3000$ & $5000-2.4 \times 10^{4}$ \\
Ref. & $(1 \mathrm{a})$ & $(2 \mathrm{a})-(2 \mathrm{~b})$ & $(3 \mathrm{a})$ & $(4 \mathrm{a})-(4 \mathrm{~b})$ & $(5 \mathrm{a})-(5 \mathrm{a}, \mathrm{b})$ \\
& & & & & \\
$n_{\mathrm{H}}{ }^{(}\left(\mathrm{cm}^{-3}\right)$ & $10^{4}$ & $10^{4}-5 \times 10^{4}$ & $5 \times 10^{4}-10^{5}$ & $10^{4}-10^{5}$ & $5 \times 10^{4}-3 \times 10^{5}$ \\
Ref. & $(1 \mathrm{a})$ & $(2 \mathrm{~b})-(2 \mathrm{a})$ & $(3 \mathrm{a})$ & $(4 \mathrm{c})-(4 \mathrm{a}, \mathrm{b}, \mathrm{d})$ & $(5 \mathrm{~b}, \mathrm{c}, \mathrm{e})-(5 \mathrm{e}, \mathrm{f}, \mathrm{g})$ \\
& & & & & \\
$A_{V}{ }^{c}(\mathrm{mag})$ & 10 & $\gtrsim 10$ & $(3 \mathrm{a})$ & $(4 \mathrm{a})$ & $(5 \mathrm{~h})$ \\
Ref. & $(1 \mathrm{~b})$ & $(2 \mathrm{c})$ & & & \\
\hline
\end{tabular}

${ }^{a}$ Incident FUV radiation field expressed in units $\chi$ of the Draine (1978) average interstellar radiation field.

${ }^{b}$ Proton gas density $n_{\mathrm{H}} \equiv n_{\mathrm{H}^{0}}+2 n_{\mathrm{H}_{2}}$.

${ }^{c}$ Visual extinction within the PDR inferred from sub-mm dust emission or CO observations.

References: (1a) Habart et al. (2003); (1b) Motte et al. (1998); (2a) Wyrowski et al. (1997b); (2b) Timmermann et al. (1996); (2c) Minchin et al. (1993); (3a) Jansen et al. (1994, 1995); (4a) Wyrowski et al. (1997b, 2000); (4b) Draine \& Bertoldi (1996, 2000); (4c) Black \& van Dishoeck (1987); (4d) Field et al. (1998); (5a) Marconi et al. (1998); (5b) Tielens \& Hollenbach (1985); (5c) Tielens et al. (1993); (5d) Tauber et al. (1994); (5e) Wyrowski et al. (1997a); (5f) Herrmann et al. (1997); (5g) Simon et al. (1997); (5h) Hogerheijde et al. (1995).

\begin{tabular}{|c|c|c|c|c|c|}
\hline & Oph W & S140 & IC 63 & NGC 2023 & Orion Bar \\
\hline $\begin{array}{l}\mathrm{H}_{2} 0-0 \mathrm{~S}(3)^{a} 9.66 \mu \mathrm{m} \\
\left(\mathrm{SWS}, 20^{\prime \prime}\right)\end{array}$ & $13.7[8]$ & $16.8[30]$ & $10[29]$ & $16.5[13.5]$ & $59.7[11]$ \\
\hline$T_{\mathrm{rot}}^{b}(\mathrm{~K})$ & $330 \pm 15$ & $500 \pm 40$ & $620 \pm 45$ & $330 \pm 15$ & $390 \pm 20$ \\
\hline$N_{\mathrm{H}_{2}}^{c}\left(10^{21} \mathrm{~cm}^{-2}\right)$ & 0.6 & 0.2 & 5 & 0.7 & 1 \\
\hline Ref. & (1) & (2) & (3a) & $(4 a)$ & (5a) \\
\hline $\mathrm{H}_{2} 1-0 \mathrm{~S}(1)^{a} 2.12 \mu \mathrm{m}$ & $3.1[16]$ & - & $1.84[13]$ & $7[20]$ & $13[10]$ \\
\hline Beam & $1^{\prime \prime}$ & & $74^{\prime \prime}$ & $1^{\prime \prime}$ & $1.5^{\prime \prime}$ \\
\hline $1-0 \mathrm{~S}(1) / 2-1 \mathrm{~S}(1)$ & - & - & $2.2[23]$ & $2.8[18]$ & $2.3[7]$ \\
\hline Ref. & (1) & & $(3 b)$ & $(4 b, c)$ & $(5 b, c)$ \\
\hline $\begin{array}{l}\text { PAH }(2-15 \mu \mathrm{m}) / 10^{3} \\
\left(\text { ISOCAM, } 6^{\prime \prime}\right)\end{array}$ & 3.6 & - & 0.8 & 11 & 62 \\
\hline Ref. & (1) & & (3c) & $(4 d)$ & $(5 d)$ \\
\hline
\end{tabular}

${ }^{a}$ Intensities (in $10^{-5} \mathrm{erg} \mathrm{s}^{-1} \mathrm{~cm}^{-2} \mathrm{sr}^{-1}$ ) with relative uncertainty in \% (in between brackets). $\mathrm{H}_{2}$ line intensities have not been corrected for dust attenuation. The 1-0 S(1) line intensity has been smoothed to the $\sim 20^{\prime \prime}$ beam of SWS or multiplied by a beam factor.

${ }^{b}$ Excitation temperature of $\mathrm{H}_{2}$ pure rotational levels with $J \leq 7$.

${ }^{c} \mathrm{H}_{2}$ column density inferred from the intensity of $\mathrm{H}_{2}$ rotational lines and assuming that the population distribution of low $\mathrm{H}_{2}$ rotational levels is essentially in LTE.

References: (1) Data corresponding to the peak of the $\mathrm{H}_{2}$ emission in Habart et al. (2003); (2) Timmermann et al. (1996); (3a) Thi et al. (1999) (note that the observations cannot be fitted by a single excitation temperature but two components at $\sim 100 \mathrm{~K}$ and $\sim 620 \mathrm{~K}$ are needed); (3b) Luhman et al. (1997); (3c) data from Cesarsky, priv. com.; (4a) Moutou et al. (1999); (4b) Field et al. (1998); (4c) Burton et al. (1998); (4d) Abergel et al. (2002); (5a) data from Bertoldi, priv. com.; (5b) van der Werf et al. (1996); (5c) Walmsley et al. (2000); (5d) Cesarsky et al. (2000).

discrepancy is that, in the models, the gas is not hot enough or alternatively that the column density of $\mathrm{H}_{2}$ is too low in the zones where the gas is warm. One explanation of this discrepancy is that the $\mathrm{H}_{2}$ formation rate is larger at high gas temperatures, moving the $\mathrm{H}^{0} / \mathrm{H}_{2}$ transition zone closer to the edge of the PDR. Habart et al. (2003) have shown that the observed $\mathrm{H}_{2}$ excitation from the moderately excited Oph W PDR can be accounted for by increasing the $\mathrm{H}_{2}$ formation rate by a factor about 5 (compared to the standard $\mathrm{H}_{2}$ formation rate derived in the diffuse ISM by Jura 1975) to $\sim 2 \times 10^{-16} \mathrm{~cm}^{3} \mathrm{~s}^{-1}$ at $T_{\text {gas }} \simeq$ $330 \mathrm{~K}$. In this study, we extend our study of the $\mathrm{H}_{2}$ formation in
PDRs (described in Sect. 2) spanning a wide range of excitation conditions.

\subsection{PDR model}

In order to analyse the $\mathrm{H}_{2}$ emission observations from the PDRs, we use an updated version of the stationary PDR model described in Le Bourlot et al. (1993). In this model, a PDR is represented by a semi-infinite plane-parallel slab with an incident radiation field. The input parameters are (i) the incident FUV field $\chi$, and (ii) the proton gas density $n_{\mathrm{H}}$. We will 
consider constant density models with $n_{\mathrm{H}}=10^{4}-10^{5} \mathrm{~cm}^{-3}$ and $\chi=10^{2}-10^{5}$ in the range of values prevailing in our PDR sample (see Table 1). With the inputs $\chi$ and $n_{\mathrm{H}}$, the model solves the chemical and thermal balance starting from the slab edge at each $A_{v}$-step in the cloud. The $\mathrm{H}_{2}$ abundance results from a balance between the formation of $\mathrm{H}_{2}$ on dust grains and the photodissociation of $\mathrm{H}_{2}$ by FUV flux, which is attenuated by dust extinction and self-shielding in the $\mathrm{H}_{2}$ lines. At equilibrium, the density of atomic $\left(n_{\mathrm{H}^{0}}\right)$ and molecular hydrogen $\left(n_{\mathrm{H}_{2}}\right)$ are given by

$R_{\mathrm{f}} n_{\mathrm{H}} n_{\mathrm{H}^{0}}=R_{\mathrm{d}}(0) \times \chi \mathrm{e}^{-\tau_{\mathrm{d}}} \times f_{\mathrm{s}}\left(N\left(\mathrm{H}_{2}\right)\right) \times n_{\mathrm{H}_{2}}$

with $R_{\mathrm{f}}\left(\mathrm{cm}^{3} \mathrm{~s}^{-1}\right)$ the $\mathrm{H}_{2}$ formation rate, $R_{\mathrm{d}}(0) \sim 5 \times 10^{-11} \mathrm{~s}^{-1}$ the unshielded photodissociation rate per $\mathrm{H}_{2}$ for $\chi=1, N\left(\mathrm{H}_{2}\right)$ the colum density of $\mathrm{H}_{2}$ and $e^{-\tau_{\mathrm{d}}}$ and $f_{\mathrm{s}}\left(N\left(\mathrm{H}_{2}\right)\right)$ respectively the dust extinction and the $\mathrm{H}_{2}$ self-shielding factors. In this study, we adopt $R_{\mathrm{f}}$ constant throughout the PDRs. The standard value inferred from observations of $\mathrm{H}_{2} \mathrm{UV}$ absorption lines in interstellar diffuse clouds is $R_{\mathrm{f}}^{0} \sim 3 \times 10^{-17} \mathrm{~cm}^{3} \mathrm{~s}^{-1}$ (Jura 1975). We assume that the energy released by the nascent molecule $(4.5 \mathrm{eV})$ is equally distributed between the kinetic energy of $\mathrm{H}_{2}\left(E_{\mathrm{k}}\right)$, the internal energy of $\mathrm{H}_{2}\left(E_{\text {int }}\right)$ and the internal energy of the grain $\left(E_{\text {grain }}\right)$. Moreover, we assume that the internal energy of the nascent $\mathrm{H}_{2}$ is distributed in Boltzmann distribution through the energy levels. The energy used for the heating per $\mathrm{H}_{2}$ formation is $E_{\mathrm{k}}+E_{\mathrm{int}} \times f$ with $E_{\mathrm{k}} \sim 1.5 \mathrm{eV}$ and $E_{\mathrm{int}} \times f \sim$ $0.7 \mathrm{eV}$ where $f$ is the fraction of the internal energy of the nascent $\mathrm{H}_{2}$ contributing to the heating. For the collisional excitation and de-excitation of $\mathrm{H}_{2}$, we adopt the $\mathrm{H}^{0}-\mathrm{H}_{2}$ inelastic rates of Martin \& Mandy (1995) extrapolated to low temperatures. The heating rate due to the photoelectric effect on small dust grains is derived from the formalism of Bakes \& Tielens (1994). For the physical conditions prevailing in our PDRs, the heating is mainly due to the photoelectric effect. Nevertheless, for high $R_{\mathrm{f}}\left(\sim 10 \times R_{\mathrm{f}}^{0}\right)$ the heating rate per $\mathrm{H}_{2}$ formation becomes comparable (factor of $\sim 2$ lower) to the photoelectric heating rate.

\section{2. $\mathrm{H}_{2}$ line intensity ratios as diagnostic of the $\mathrm{H}_{2}$ formation rate}

The $(v, J)$ excited states of $\mathrm{H}_{2}$ can be populated by inelastic collisions with gas phase species, UV pumping and by the formation process. The population distribution of $\mathrm{H}_{2}$ levels is a function of the gas density, the gas temperature and the UV flux. Hence, the $\mathrm{H}_{2}$ line intensity ratios - which depend on the physical conditions in the photodissociation front where atomic hydrogen becomes molecular - should probe the $\mathrm{H}_{2}$ formation rate which controls the location of the $\mathrm{H}^{0} / \mathrm{H}_{2}$ transition zone in PDRs. In the following, we study the dependence of several $\mathrm{H}_{2}$ line ratios as a function of $R_{\mathrm{f}}$ (predicted by model calculations).

In Fig. 1, we show the model predictions (face-on) for the intensity of the 1-0 S(1) line as well as for several commonly observed line intensity ratios as a function of the molecular hydrogen formation rate. First, we discuss the dependence of the 1-0 S(1) line intensity on $R_{\mathrm{f}}$. At equilibrium (Eq. (1)), the intensity of the $1-0 \mathrm{~S}(1)$ line $I_{\mathrm{H}_{2}}^{\mathrm{f}}$ is proportional to $R_{\mathrm{f}} n_{\mathrm{H}} N\left(\mathrm{H}^{0}\right)$ where $N\left(\mathrm{H}^{0}\right)$ is the column density of atomic $\mathrm{H}$ atoms. For high values of $\chi / n_{\mathrm{H}}(\gtrsim 0.01)$, the $\mathrm{H}^{0} / \mathrm{H}_{2}$ transition is driven by the dust opacity and $N\left(\mathrm{H}^{0}\right)$ is a constant equal to a few $10^{21} \mathrm{~cm}^{-2}$. We thus have $I_{\mathrm{H}_{2}}^{\mathrm{f}} \propto R_{\mathrm{f}} n_{\mathrm{H}}$ (see Fig. 1a). Conversely, when $\chi / n_{\mathrm{H}} \lesssim 0.01$, molecular hydrogen self shields sufficiently that the gas goes molecular before the dust gets optically thick. In this case, using the approximation $f_{\mathrm{s}}\left(N\left(\mathrm{H}_{2}\right)\right)=\left(N\left(\mathrm{H}_{2}\right) / N_{0}\right)^{-3 / 4}$ with $N_{0}=10^{14} \mathrm{~cm}^{-2}$ (Draine \& Bertoldi 1996) for $N\left(\mathrm{H}_{2}\right) \lesssim$ $10^{21} \mathrm{~cm}^{-2}$, we find $R_{\mathrm{f}} n_{\mathrm{H}} N\left(\mathrm{H}^{0}\right)=4 R_{\mathrm{d}}(0) \times \chi \times N\left(\mathrm{H}_{2}\right)^{1 / 4} N_{0}^{3 / 4}$ (Hollenbach \& Tielens 1999). At the $\mathrm{H}^{0} / \mathrm{H}_{2}$ transition, we have $N\left(\mathrm{H}_{2}\right)=N(\mathrm{H}) / 2$ with $N(\mathrm{H})$ the proton column density from the edge of the PDR to the $\mathrm{H}^{0} / \mathrm{H}_{2}$ transition zone. Finally, $I_{\mathrm{H}_{2}}^{\mathrm{f}} \propto \chi \times N(\mathrm{H})^{1 / 4}$ (see Fig. 1a).

The low-J rotational $v=0-0 \mathrm{H}_{2}$ lines have a somewhat different dependence upon $R_{\mathrm{f}}$ because at densities above $10^{4} \mathrm{~cm}^{-3}$ of interest to us, their population distribution is essentially in LTE and the line intensities depend mainly on the temperature in the photodissociation front where hydrogen becomes molecular. In the range of $\chi$ and $n_{\mathrm{H}}$ studied here, the gas temperature profiles as a function of depth in the PDR are fairly insensitive to the fraction of molecular hydrogen (heating is mainly due to photoelectric emission from dust grains and cooling to fine structure line emission of $\mathrm{O}^{0}$ and $\mathrm{C}^{+}$) but higher $\mathrm{H}_{2}$ formation rates cause the photodissociation front to shift closer to the surface where the temperature is higher. We show in Figs. $1 \mathrm{~b}$ and $\mathrm{d}$ the dependence of the $0-0 \mathrm{~S}(1) / 0-0 \mathrm{~S}(3)$ and $0-0 \mathrm{~S}(3) / 1-0 \mathrm{~S}(1)$ line ratios as functions of $R_{\mathrm{f}}$. The ratio of the two $0-0$ lines is essentially a measure of temperature and thus decreases as $R_{\mathrm{f}}$ increases as a consequence of the temperature increase in the dissociation front. This effect also causes a sharper increase in $0-0 \mathrm{~S}(3)$ than in $1-0 \mathrm{~S}(1)$ and thus the ratio $0-0 \mathrm{~S}(3) / 1-0 \mathrm{~S}(1)$ increases with increasing $R_{\mathrm{f}}$.

We also show in Fig. 1c the dependence of the 2-1 S(1)/1-0 S(1) line predicted by the models as a function of $R_{\mathrm{f}} / R_{\mathrm{f}}^{0}$. This commonly observed line ratio is known in PDRs to vary between values of $\sim 2$ typical of pure fluorescence to values of $\sim 3-5$ at high densities (above $10^{5} \mathrm{~cm}^{-3}$ ) when collisional deexcitation of higher vibrational levels becomes competitive with radiative decay (Black \& van Dishoeck 1987; Draine \& Bertoldi 1996). One sees from Fig. 1c that the model predicted line ratio for $\chi \leq 10^{3}$ and $n_{\mathrm{H}} \leq 10^{5} \mathrm{~cm}^{-3}$ is independent of $R_{\mathrm{f}}$ (as well as of $\chi$ and $n_{\mathrm{H}}$ ) and equal to $\sim 2$. This is due to the fact that the densities and radiation fields of interest to us (and consequently assumed in the models) are in the range for which the population of excited vibrational levels of $\mathrm{H}_{2}$ are controlled by purely radiative cascade process.

In summary, we find that the $0-0 \mathrm{~S}(3) / 1-0 \mathrm{~S}(1)$ line intensity ratio depends considerably on the $\mathrm{H}_{2}$ formation rate via the variation of both the gas temperature and the UV flux in the $\mathrm{H}^{0} / \mathrm{H}_{2}$ transition zone. However, the changes in this ratio can also be due to changes in the radiation field and density. Thus, by comparing this $\mathrm{H}_{2}$ line intensity ratio predicted by the model (essentially independent of the geometry and of the total column density) with observations from PDRs where $\chi$ and $n_{\mathrm{H}}$ have been determined from other observations, we can expect to probe the $\mathrm{H}_{2}$ formation rate in PDRs.

In Fig. 2, we show the $\mathrm{H}_{2} 0-0 \mathrm{~S}(3) / 1-0 \mathrm{~S}(1)$ line intensity ratio as predicted by the model for an edge-on geometry 
(a)

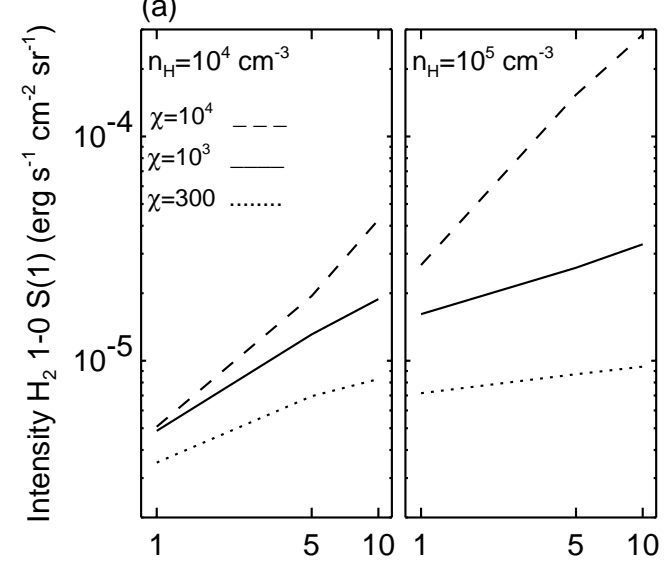

(c)

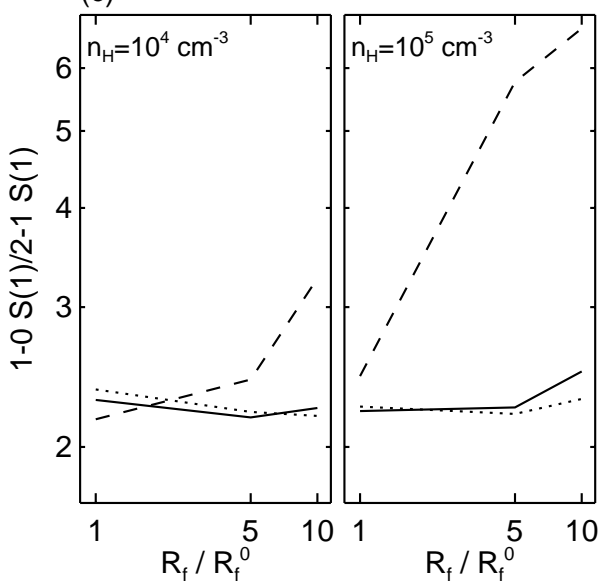

(b)

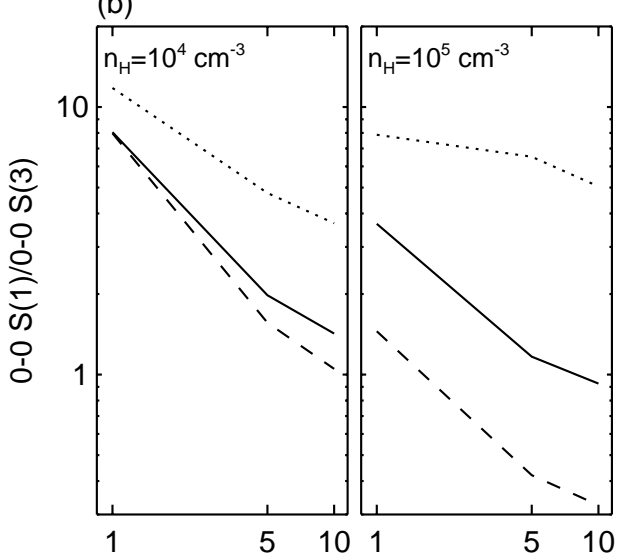

(d)

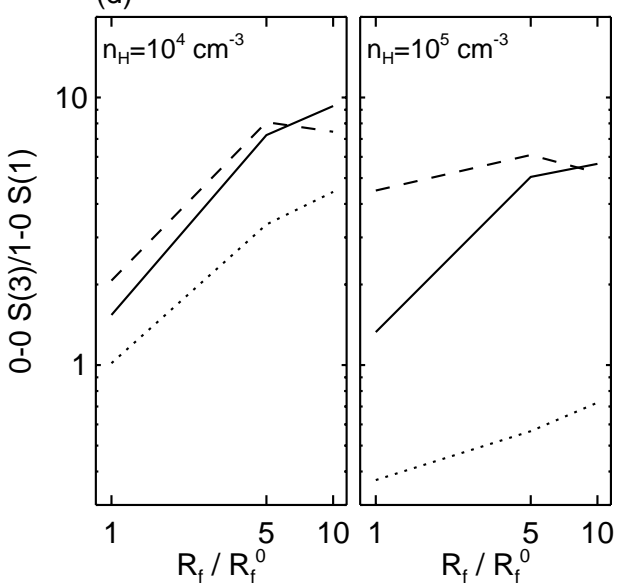

Fig. 1. Panel a): Intensity of the $\mathrm{H}_{2}$ 1-0 S(1) line emission predicted by models (face-on) for two gas densities and different incident radiation fields $-\chi=300$ (dotted lines), $\chi=10^{3}$ (solid lines) and $\chi=10^{4}$ (dashed lines) - as a function of the value of $\mathrm{H}_{2}$ formation rate divided by $R_{\mathrm{f}}^{0}$ corresponding to the formation rate based upon Copernicus data (Jura 1975). Panels b), c) and d): several $\mathrm{H}_{2}$ line intensity ratios for the same models.

(as most of our PDRs are, see references in Table 1) in the $\mathrm{H}_{2}$ emission region as a function of $\chi$ and for different $\mathrm{H}_{2}$ formation rates. We compare these predicted line intensity ratios with observational data for our PDRs sample described in Sect. 2. The data has been corrected for dust attenuation applying a correction factor $\tau /\left(1-\mathrm{e}^{-\tau}\right)$. Here, we use the extinction curve of Draine (1989) and the visual extinction within the PDR reported in Table 1 derived from sub-mm dust emission or $\mathrm{CO}$ observations. This assumes that our PDRs are exactly planar and edge-on and that densities are similar in the $\mathrm{H}_{2}$ emission layer and in the cold molecular layer which is questionable because the column density inferred from the molecular hydrogen rotational transitions is generally an order of magnitude lower than the value derived from sub-mm dust emission or CO observations. The explanation of this difference is not clear. It could occur in an edge-on geometry due to beam dilution effects (i.e., $\mathrm{H}_{2}$ emission region not resolved by ISO-SWS) or because of density gradients. For reasons of simplicity, we have adopted in Fig. 2 the estimate inferred from sub-mm dust emission or CO observations. The error involved here is small since using the column density derived from the molecular hydrogen rotational transitions, the $\mathrm{H}_{2} \quad 0-0 \mathrm{~S}(3) / 1-0 \mathrm{~S}(1)$ line intensity ratio would diminish by $\sim 30 \%$ which is comparable to the error bars.
In the following, we discuss for each PDR of our sample the value of $R_{\mathrm{f}}$ required to account for the observed $\mathrm{H}_{2}$ excitation. For Oph W $\left(\chi \sim 250, n_{\mathrm{H}} \sim 10^{4} \mathrm{~cm}^{-3}\right)$ and IC $63(\chi \sim$ $\left.650, n_{\mathrm{H}} \sim 5 \times 10^{4}-10^{5} \mathrm{~cm}^{-3}\right)$, we find that models with a high $\mathrm{H}_{2}$ formation rate (i.e., $R_{\mathrm{f}} \gtrsim 5 \times R_{\mathrm{f}}^{0}$ ) roughly reproduce the $0-0 \mathrm{~S}(3) / 1-0 \mathrm{~S}(1)$ line ratio observed. For the standard $\mathrm{H}_{2}$ formation rate, the $0-0 \mathrm{~S}(3) / 1-0 \mathrm{~S}(1)$ line ratio is underestimated by a factor about 4 . For the more highly excited PDRs, i.e., NGC $2023\left(\chi \sim 500-3000, n_{\mathrm{H}} \sim 10^{4}-10^{5} \mathrm{~cm}^{-3}\right)$ and the Orion Bar $\left(\chi \sim 0.5-2.5 \times 10^{4}, n_{\mathrm{H}} \sim 5 \times 10^{4}-3 \times 10^{5} \mathrm{~cm}^{-3}\right)$, we find on the contrary that models with the standard $\mathrm{H}_{2}$ formation rate roughly match the data. In the case of S140, where we have no measurement of the intensity for the 1-0 S(1) line, we use ISO observations of the $1-0 \mathrm{Q}(3)$ line from Draine \& Bertoldi (1999) to determine the $\mathrm{H}_{2}$ rotational to rovibrational line intensity ratio. For the physical conditions of interest to us, the models predict that the $1-0 \mathrm{~S}(1) / 1-0 \mathrm{Q}(3)$ line intensity ratio is about a factor of 1.4. Applying this factor, we find that the $0-0 \mathrm{~S}(3) / 1-0 \mathrm{~S}(1)$ line intensity ratio (corrected for dust attenuation) is about 3 . Then, one sees from Fig. 2 that for $\mathrm{S} 140$ $\left(\chi \sim 100-250, n_{\mathrm{H}} \sim 10^{4}-5 \times 10^{4} \mathrm{~cm}^{-3}\right)$ models with a high $\mathrm{H}_{2}$ formation rate are - as for the moderately excited PDR Oph W and IC 63 - required to explain the observed $\mathrm{H}_{2}$ line intensity ratio. In Table 3 , we give for each region the values 

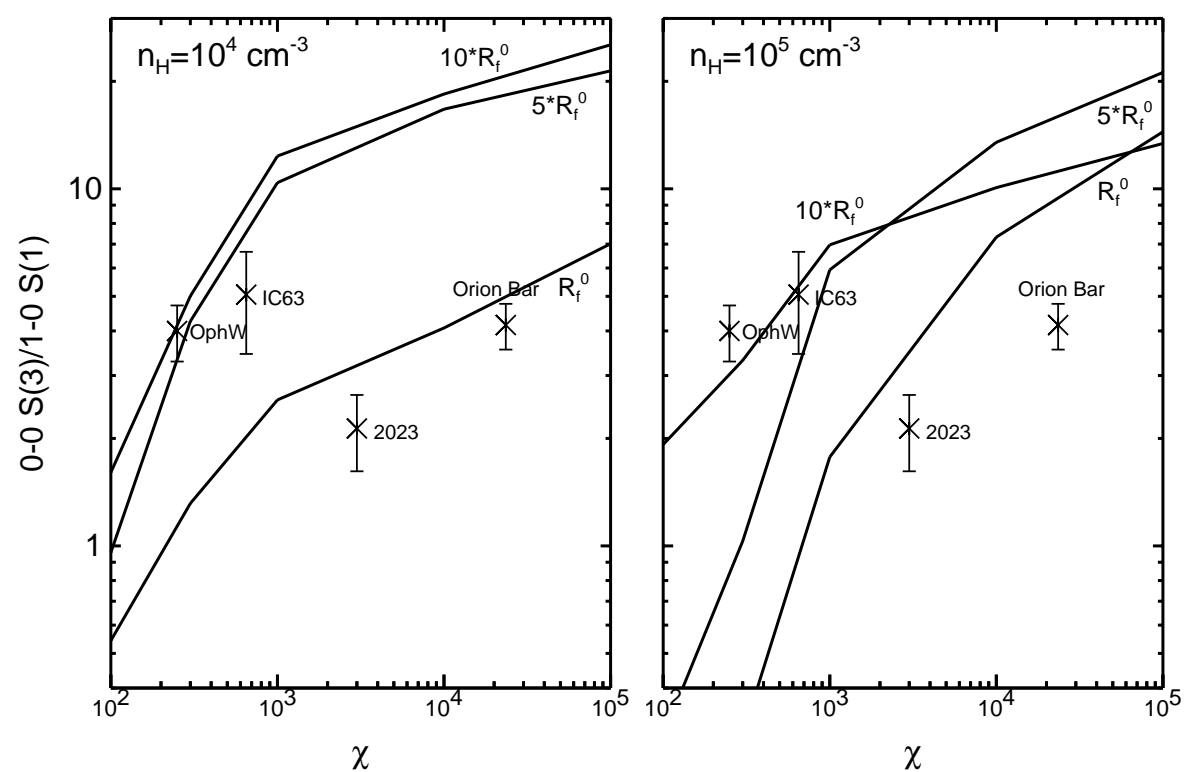

Fig. 2. $\mathrm{H}_{2}$ 0-0 S(3)/1-0 S(1) line intensity ratio, as predicted by models (edge-on) and as observed for our PDR sample (crosses with error bars), as a function of $\chi$ the FUV incident radiation field. Predicted line intensity ratios (taken in the $\mathrm{H}_{2}$ emitting zone) are presented for two gas densities and shown for three different $\mathrm{H}_{2}$ formation rates $\left(R_{\mathrm{f}}^{0}, 5 \times R_{\mathrm{f}}^{0}, 10 \times R_{\mathrm{f}}^{0}\right)$. Observed line intensity ratios have been corrected for dust attenuation (see text). For each PDR of our sample, $\chi$ has been derived from the expected FUV luminosity of the exciting star and assuming that the distance of the exciting star to the PDR is equal to the distance projected onto the sky (see Sect. 2). For S140, where we have no measurement of the intensity for the 1-0 S(1) line, we use observations of the $1-0 \mathrm{Q}(3)$ line to determine the $\mathrm{H}_{2}$ line intensity ratio (see text).
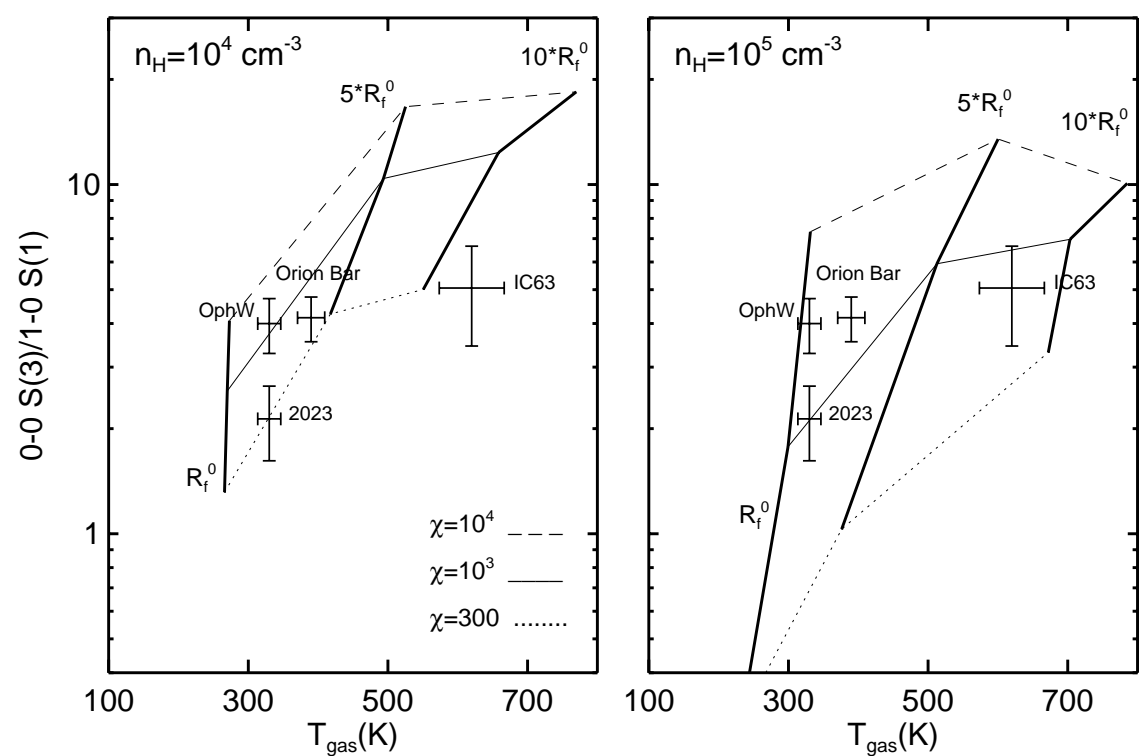

Fig. 3. $\mathrm{H}_{2} 0-0 \mathrm{~S}(3) / 1-0 \mathrm{~S}(1)$ line intensity ratio as predicted by the same models shown in Fig. 2 and as observed for our PDR sample (crosses with error bars) as a function of $T_{\text {gas }}$ the gas temperature in the $\mathrm{H}_{2}$ emitting region. For the observations, we use the excitation temperature of the $\mathrm{H}_{2}$ pure rotational levels (see text). For IC 63 , the observed $\mathrm{H}_{2}$ pure rotational lines intensity cannot be fitted by a single excitation temperature but two components at $\sim 100 \mathrm{~K}$ and $\sim 600 \mathrm{~K}$ are needed.

of the $\mathrm{H}_{2}$ formation rate derived using Fig. 2 and assuming that the gas density in the $\mathrm{H}_{2}$ emission region is about $10^{4} \mathrm{~cm}^{-3}$ for all sources. Considering that the gas density is about $10^{5} \mathrm{~cm}^{-3}$, $R_{\mathrm{f}}$ required to explain the data would be (by a factor of $\sim 2$ ) higher and lower for respectively the moderately and the highly excited PDRs. We emphasize that our determination of $R_{\mathrm{f}}$ from the $\mathrm{H}_{2}$ line intensity ratios depends on the treatment in the model of the thermal balances and the chemistry. In particular, the calculation of the grain photoelectric heating which mainly determines $T_{\text {gas }}$ in the PDR modelling (see Sect. 3.1) could affect the excitation of the lowest rotational levels $(J \leq 5)$ of $\mathrm{H}_{2}$. For example, Habart et al. (2003) have shown that, throughout the Oph W PDR, increasing the photoelectric heating rate by $\sim 50 \%$, which leads to higher $T_{\text {gas }}$ in the $\mathrm{H}^{0} / \mathrm{H}_{2}$ transition, the $0-0 \mathrm{~S}(3)$ line intensity is enhanced (by a factor $\sim 2$ ) and $R_{\mathrm{f}}$ required to explain the data would be reduced by a factor $\sim 1.5$ (see Fig. 2). However, even if we cannot precisely determine the uncertainties on our estimates of $R_{\mathrm{f}}$, the values of $R_{\mathrm{f}}$ derived here should be at most uncertain by a factor of about 2 . 
One check of our estimates of $R_{\mathrm{f}}$ is to see if the predicted $T_{\text {gas }}$ values in the $\mathrm{H}_{2}$ emitting region are consistent with the observed rotational temperatures reported in Table 1. In Fig. 3, we show the $0-0 S(3) / 1-0 S(1)$ line intensity ratio predicted by the same models shown in Fig. 2 as a function of the gas temperature in the $\mathrm{H}_{2}$ emitting region and compared with observational data. For observations, we use the excitation temperature of $\mathrm{H}_{2}$ pure rotational levels (with $J \leq 7$ ) which in principle is an upper limit as UV pumping could contribute to the excitation of $\mathrm{H}_{2}$ even for low energy levels. In the case of IC 63 where the measured pure $\mathrm{H}_{2}$ rotational lines show two excitation temperatures (at $\sim 100 \mathrm{~K}$ and $\sim 600 \mathrm{~K}$, Thi et al. 1999) there is direct evidence for such contamination. Using Fig. 3, we favour (as previously) for the moderately excited PDRs models with high $\mathrm{H}_{2}$ formation rate which can in fact explain the observed rotation temperatures. Models with a standard $\mathrm{H}_{2}$ formation rate predict in fact a gas temperature $(\leq 300 \mathrm{~K})$ lower than observed ( 300-600 K). For the Orion Bar and NGC 2023, we find on the contrary that models with standard $\mathrm{H}_{2}$ formation rate predict temperatures consistent with the data $(\sim 300-400 \mathrm{~K})$. Finally, we have checked that these models reproduce the observed absolute intensities of the $0-0 \mathrm{~S}(3)$ and 1-0 S(1) $\mathrm{H}_{2}$ lines. Taking into account the inclinations of the PDRs of our sample, which are seen edge-on except for IC 63, we find an agreement within $\lesssim 50 \%$.

It must be emphasized that these results are based on two fundamental assumptions. Firstly, we assume a uniform homogeneous gas density while PDRs may have a density gradient and could be clumpy. Nevertheless, because the modelling of the $\mathrm{H}_{2}$ excitation mainly depends upon the average gas density in the $\mathrm{H}_{2}$ emission zone, density structure effects are probably minor. In fact, the detailed study of Habart et al. (2003) of the $\mathrm{H}_{2}$ excitation toward Oph W taking into account the gas density profile reaches the same estimation of $R_{\mathrm{f}}$ deduced here.

Secondly, we assumed a static, equilibrium PDR. In reality, the propagation of the ionization and photodissociation fronts will bring fresh $\mathrm{H}_{2}$ into the zone emitting line radiation. Note that a non-equilibrium ortho-to-para $\mathrm{H}_{2}$ ratio has been observed in ISO-SWS observations towards Oph W (Habart et al. 2003), NGC 2023 (Moutou et al. 1999) and NGC 7023 (Fuente et al. 1999). Nevertheless, the model of non-equilibrium PDRs of Störzer \& Hollenbach (1998) predicts that, for the physical conditions prevailing in the Orion Bar and for an advection velocity of the order of $1 \mathrm{~km} \mathrm{~s}^{-1}$, the $\mathrm{H}_{2} 0-0 \mathrm{~S}(3) / 1-0 \mathrm{~S}(1)$ line intensity ratio varies by a factor less than $\sim 2$ relative to the steady state value.

In the next section, we review other observational constraints on the $\mathrm{H}_{2}$ formation rate based on $\mathrm{H}_{2} \mathrm{UV}$ absorption measurements.

\subsection{Other observational constraints on the $\mathrm{H}_{2}$ formation rate}

UV absorption measurements of $\mathrm{H}_{2}$ have been often used to study $\mathrm{H}_{2}$ formation, destruction, and excitation in the diffuse ISM. With the numerous new $\mathrm{H}_{2} \mathrm{UV}$ absorption line observations obtained recently by FUSE (Snow et al. 2000; Shull et al. 2000; Rachford et al. 2001, 2002; Tumlinson et al. 2002; Sonnentrucker et al. 2002), the $\mathrm{H}_{2}$ formation in the diffuse ISM has been re-considered. In particular, Gry et al. (2002) have determined the $\mathrm{H}_{2}$ formation rates over three lines of sight in the Chamaeleon clouds. They find a rate roughly constant and equal to about $4 \times 10^{-17} \mathrm{~cm}^{3} \mathrm{~s}^{-1}$ (with an uncertainty of about a factor of 2) for $n_{\mathrm{H}} \sim 30-50 \mathrm{~cm}^{-3}$ and $T_{\text {gas }} \sim 60 \mathrm{~K}$, in agreement with the rate inferred by Jura (1975). Moreover, due to the high FUSE sensitivity, fainter stars with higher extinctions could be observed with far-UV instruments (Moos et al. 2000), thus allowing the study of translucent clouds. Rachford et al. (2002), studying correlations of the $\mathrm{H}_{2}$-related quantities with the column densities of other molecules and dust extinction properties for lines of sight with $A_{v} \gtrsim 1$, investigate the dependence of the $\mathrm{H}_{2}$ formation rate with the composition and physical state of the gas and grains. Furthermore, the FUSE observations in the lower metallicity environments of the Small and Large Magellanic Clouds allow us to probe $\mathrm{H}_{2}$ formation and destruction in physical and chemical environments different from the Galaxy. Tumlinson et al. (2002) find that to reproduce the reduced molecular fraction and enhanced rotational excitation in the SMC and LMC, a low $\mathrm{H}_{2}$ formation rate $\left(R_{\mathrm{f}} \sim 3 \times 10^{-18} \mathrm{~cm}^{3} \mathrm{~s}^{-1}\right)$ and a high UV field relative to diffuse Galactic medium are required.

Combining the FUSE results in the diffuse Chamaeleon clouds and the ISO observations discussed above, we find that for a wide range of physical conditions $-1 \lesssim \chi \lesssim 10^{4}$, $100 \mathrm{~cm}^{-3} \lesssim n_{\mathrm{H}} \lesssim 10^{5} \mathrm{~cm}^{-3}, 50 \mathrm{~K} \lesssim T_{\text {gas }} \lesssim 600 \mathrm{~K}, 10 \mathrm{~K} \lesssim$ $T_{\text {dust }} \lesssim 100 \mathrm{~K}$ (see Tables 1 and 3 ) $-\mathrm{H}_{2}$ forms efficiently $\left(R_{\mathrm{f}} \sim 4 \times 10^{-17}-1.5 \times 10^{-16} \mathrm{~cm}^{3} \mathrm{~s}^{-1}\right)$. This result raises questions about our understanding of the $\mathrm{H}_{2}$ formation process. In Sect. 5, we re-examine models of $\mathrm{H}_{2}$ formation with this in mind and compare in Sect. 6 the observational constraints on the $\mathrm{H}_{2}$ formation rate with model predictions.

\section{Influence of aromatic dust on the $\mathrm{H}_{2}$ formation process in PDRs}

In PDRs, small dust grains are intimately coupled to the evolution of the gas. In fact, recent theoretical (Bakes \& Tielens 1994; Weingartner \& Draine 2001) and observational (Habart et al. 2001) work has shown that small grains (radius $\leq 100 \AA$ ) dominate the photoelectric heating. Furthermore, given that small grains make a dominant contribution to the total grain surface (see Sect. 6), it is plausible that they play a dominant role in $\mathrm{H}_{2}$ formation. Therefore, if $\mathrm{H}_{2}$ forms on small grains, we can expect that both the grain photoelectric heating rate and the $\mathrm{H}_{2}$ formation rate will scale with their abundance. In particular, increasing their abundance would lead to higher gas temperature and would bring the $\mathrm{H}^{0} / \mathrm{H}_{2}$ transition closer to the edge: this could significantly enhance the rotational and fluorescent emission of $\mathrm{H}_{2}$. Habart et al. (2003) have shown that, assuming that $R_{\mathrm{f}}$ scales with the PAH abundance, the enhancement in $R_{\mathrm{f}}$ required to account for the observed $\mathrm{H}_{2}$ emission from $\mathrm{Oph} \mathrm{W}$ may result from an increased PAH abundance. Based on this, we investigate what we can predict concerning the influence of PAHs on the $\mathrm{H}_{2}$ formation process. 


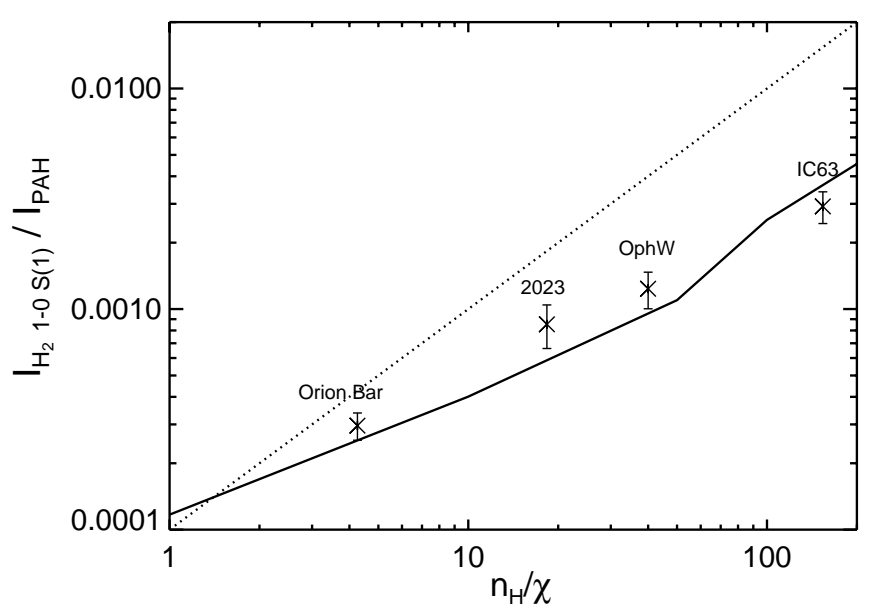

Fig. 4. Ratio between the $\mathrm{H}_{2} 1-0 \mathrm{~S}(1)$ line and PAH emission as predicted by edge-on model (solid line) and as observed (crosses with error bars) as a function of $n_{\mathrm{H}} / \chi$. The dotted straight lines shows the linear dependence of the emission ratio with $n_{\mathrm{H}} / \chi$ expected from Eq. (2) when $\chi / n_{\mathrm{H}} \gtrsim 0.01$. For the PDRs of our sample, $n_{\mathrm{H}} / \chi$ has been taken equal to the ratio between the average value of the $n_{\mathrm{H}}$ determinations given in Table 1 and $\chi$ determined from the expected FUV luminosity of the exciting star and assuming that the distance of the exciting star to the PDR is equal to the distance projected onto the sky (see Sect. 2).

To free oneself of the influence of the PAHs on the thermal balance, we study here the ratio between the $\mathrm{H}_{2} 1-0 \mathrm{~S}(1)$ line (whose intensity does not depend on $T_{\text {gas }}$, see Sect. 3.2) and the PAH emission. We first express analytically the $\mathrm{H}_{2}$ fluorescent to PAH emission ratio $I_{\mathrm{H}_{2}}^{\mathrm{f}} / I_{\mathrm{PAH}}$ as a function of the $\mathrm{H}_{2}$ formation rate and the $\mathrm{PAH}$ abundance, as well as the physical conditions $\left(n_{\mathrm{H}}, \chi\right)$, in order to examine qualitatively the dependence of this ratio with these parameters. In Sect. 3.2, we have seen that the $\mathrm{H}_{2} 1-0 \mathrm{~S}(1)$ line emission $I_{\mathrm{H}_{2}}^{\mathrm{f}}$ goes roughly as (i) $R_{\mathrm{f}} n_{\mathrm{H}}$ for high $\chi / n_{\mathrm{H}}$ and as (ii) $\chi$ for low $\chi / n_{\mathrm{H}}$. The emission of aromatic dust scales with the intensity of the FUV radiation field (Puget et al. 1985; Sellgren et al. 1985) and we have $I_{\mathrm{PAH}} \propto \chi \mathrm{e}^{-\tau_{\mathrm{d}}} \times N_{\mathrm{H}}[\mathrm{C} / \mathrm{H}]_{\mathrm{PAH}}$ with $[\mathrm{C} / \mathrm{H}]_{\mathrm{PAH}}$ the abundance of carbon locked up in PAHs. As the column density over which PAHs emits is a few $10^{21} \mathrm{~cm}^{-2}$ (where dust is optically thin), $I_{\mathrm{PAH}}$ goes as $\chi \times[\mathrm{C} / \mathrm{H}]_{\mathrm{PAH}}$. From these considerations, we find

$$
\frac{I_{\mathrm{H}_{2}}^{\mathrm{f}}}{I_{\mathrm{PAH}}} \propto\left\{\begin{aligned}
\frac{R_{\mathrm{f}}}{[\mathrm{C} / \mathrm{H}]_{\mathrm{PAH}}} \times \frac{n_{\mathrm{H}}}{\chi} & : \quad \chi / n_{\mathrm{H}} \gtrsim 0.01 \\
\frac{1}{[\mathrm{C} / \mathrm{H}]_{\mathrm{PAH}}} & : \quad \chi / n_{\mathrm{H}} \lesssim 0.01 .
\end{aligned}\right.
$$

Thus, by studying the observed $\mathrm{H}_{2}$ fluorescent to PAH emission ratio from PDRs covering a wide range of $n_{\mathrm{H}} / \chi$ ratio, we should be able to probe the $\mathrm{H}_{2}$ formation on PAH: if $\mathrm{H}_{2}$ forms on PAHs, we expect that the $I_{\mathrm{H}_{2}}^{\mathrm{f}} / I_{\mathrm{PAH}}$ ratio scales with $n_{\mathrm{H}} / \chi$. In other words, the $R_{\mathrm{f}} /[\mathrm{C} / \mathrm{H}]_{\mathrm{PAH}}$ ratio is constant and does not vary from one region to another.

We now compare the ratio between the $\mathrm{H}_{2}$ 1-0 S(1) line and PAH emission observed from the PDRs of our sample to model results. For the models, we consider $\chi=10^{2}-10^{4}$ and $n_{\mathrm{H}}=10^{4}-10^{5} \mathrm{~cm}^{-3}$ which corresponds to the range of values prevailing in our PDR sample (see Table 1). We adopt a constant $R_{\mathrm{f}} /[\mathrm{C} / \mathrm{H}]_{\mathrm{PAH}}$ ratio corresponding to the values derived in the Oph W PDR, i.e., $R_{\mathrm{f}} \simeq 5 \times R_{\mathrm{f}}^{0}$ and $[\mathrm{C} / \mathrm{H}]_{\mathrm{PAH}} \simeq 0.5 \times 10^{-4}$ (Habart et al. 2003). The power emitted by PAHs has been derived from the PAH absorption cross-section of Verstraete \& Léger (1992) and the size distribution described in Sect. 6. In Fig. 4, we show the $\mathrm{H}_{2} 1-0 \mathrm{~S}(1) / \mathrm{PAH}$ emission ratio predicted by the model in the $\mathrm{H}_{2}$ emission zone as a function of the $n_{\mathrm{H}} / \chi$ ratio. As expected from the Eq. (2), for $\chi / n_{\mathrm{H}} \gtrsim 0.01$ the $\mathrm{H}_{2} 1-0 \mathrm{~S}(1) / \mathrm{PAH}$ emission ratio increases proportionally with $n_{\mathrm{H}} / \chi$.

The observational ratios for our PDR sample are compared to these predictions. The observed points roughly fall on the model curve. From one PDR to another, the change of the emission ratio seems to result mainly from the physical conditions (i.e., $n_{\mathrm{H}} / \chi$ ) variations. We deduce from this that the $R_{\mathrm{f}} /[\mathrm{C} / \mathrm{H}]_{\mathrm{PAH}}$ ratio is roughly constant which suggests that formation of $\mathrm{H}_{2}$ on PAHs should be important. However, the correlation between $R_{\mathrm{f}}$ and $[\mathrm{C} / \mathrm{H}]_{\mathrm{PAH}}$ found in our PDR sample is observed not to apply to the main PDR in the 30 Dor star forming region in the Large Magellanic Cloud where for $n_{\mathrm{H}} / \chi \sim 1$ the $\mathrm{H}_{2} 1-0 \mathrm{~S}(1)$ line to PAH emission ratio is measured to be $\sim 0.001$ (Boulanger et al. 2003). In future work, this comparison should be extended so as to include all small grains, i.e., not only the band carriers but also the very small grains emitting at longer wavelengths.

\section{5. $\mathrm{H}_{2}$ formation mechanisms on grain surfaces}

In this section, with the aim of explaining our observational constraints on the $\mathrm{H}_{2}$ formation, we examine models of $\mathrm{H}_{2}$ formation mechanism. Two general mechanisms for forming $\mathrm{H}_{2}$ on grain surfaces have generally been proposed:

1. formation by physisorbed $\mathrm{H}$ atoms (LangmuirHinshelwood) whereby two adsorbed and mobile physisorbed $\mathrm{H}$ atoms (bound simply via van der Waals interaction with binding energies of the order of $0.05 \mathrm{eV}$ or $500 \mathrm{~K}$, Katz et al. 1999) interact to recombine and desorb as $\mathrm{H}_{2}$ and

2. formation by the interaction of an $\mathrm{H}$ atom from the gas phase with a chemisorbed $\mathrm{H}$ atom (with binding energy roughly $1 \mathrm{eV}$ equivalent to $\sim 10000 \mathrm{~K}$, Fromherz et al. 1993) forming desorbed $\mathrm{H}_{2}$ (the Eley-Rideal mechanism).

In the following discussion, we describe these processes mainly focusing on the version of the Langmuir-Hinshelwood mechanism whereby one of the adsorbed $\mathrm{H}$-atoms is originally in a chemisorbed site (Hollenbach \& Salpeter 1971; Cazaux \& Tielens 2002, 2003). This approach will be called in the following the indirect chemisorption approach as opposed to the direct chemisorption or Eley-Rideal mechanism. We make simple empirical estimates of rates for these processes as a function of the physical conditions and grain characteristics. We consider small grains (SGs), dust particles with radii of a few $\AA$ to $100 \AA$ (i.e., PAHs and Very Small Grains, VSGs) which fluctuate in temperature in the radiation field, and big grains (BGs), larger grains of radii $>0.01 \mu \mathrm{m}$ in thermal equilibrium with the radiation field (Désert et al. 1990). 


\subsection{The Langmuir-Hinshelwood mechanism}

In the formation from physisorbed atoms (pure LangmuirHinshelwood), an $\mathrm{H}$ atom is already physisorbed on the grain surface. A second $\mathrm{H}$ atom from the gas sticks to the grain and diffuses to finally recombine with the first $\mathrm{H}$ atom to form $\mathrm{H}_{2}$. The rate depends upon the competition between the mobility of the $\mathrm{H}$ atom on the grain surface and its thermal evaporation rate. In the laboratory, this process is observed to be efficient on grains with $7 \lesssim T_{\text {dust }} \lesssim 20 \mathrm{~K}$ (Pirronello et al. 1997, 1999).

Amongst the dust grain populations described above, only BGs could carry a large fraction of physisorbed $\mathrm{H}$ atoms. Indeed, after a UV photon is absorbed by SGs, the physisorbed $\mathrm{H}$ atoms should evaporate. Further, for the physical conditions typical of the PDRs, the rate of thermal fluctuations $\tau_{\text {abs }}^{-1}$ $\left(\sim 1.6 \times 10^{-9} \times N(\mathrm{C}) \times \chi \mathrm{s}^{-1}\right.$ with $N(\mathrm{C})$ the number of carbon atom in the grain, Verstraete et al. 2001) is comparable to or larger than the accretion rate of $\mathrm{H}$ atoms $\tau_{\mathrm{acc}}^{-1}$ : for a grain with a radius of $1.5 \mathrm{~nm}(N(\mathrm{C}) \simeq 1600)$, we find $\tau_{\text {abs }}^{-1} / \tau_{\text {acc }}^{-1} \sim$ $1000 \times\left(100 \mathrm{~K} / T_{\text {gas }}\right)^{0.5} \times \chi / n_{\mathrm{H}}$. Thus for $\chi / n_{\mathrm{H}}$ larger than 0.001 (true in general for the objects in Table 1), UV radiation in PDRs will keep SG surfaces clean.

Since this mechanism will only be efficient on BGs and in interstellar regions of low excitation (in PDRs we find typically $T_{\mathrm{BGs}} \geq 30 \mathrm{~K}$, see Table 3 ), we neglect it in what follows.

\subsection{Formation involving a chemisorbed $\mathrm{H}$ atom}

We now consider the case where the first $\mathrm{H}$ atom is bound to the surface in a chemisorbed site. The second $\mathrm{H}$ atom from the gas phase reaches this site directly (direct chemisorption or EleyRideal, Duley 1996; Parneix \& Brechignac 1998) or after diffusion on the grain surface (indirect chemisorption, Hollenbach \& Salpeter 1971; Cazaux \& Tielens 2002, 2003). The incident $\mathrm{H}$ atom crosses the activation barrier $E_{\mathrm{a}}$ (typically about 200-1500 K, Fromherz et al. 1993; Parneix \& Brechignac 1998; Sidis et al. 2000; Cazaux \& Tielens 2003) by either thermal hopping or tunneling and recombines to finally form $\mathrm{H}_{2}$. This process involving strongly bound $\mathrm{H}$ atoms will be efficient at higher dust surface temperatures than the pure LangmuirHinshelwood mechanism.

We first consider the direct Eley-Rideal mechanism. This involves reaction of the incoming $\mathrm{H}$ atom with an chemisorbed $\mathrm{H}$ on the grain surface without any requirement for adsorption and thermal accommodation. The formation probability $P_{\mathrm{H}_{2}}$ can be written as:

$P_{\mathrm{H}_{2}}=f \times \eta$

with $f$ the probability that the incident $\mathrm{H}$ atom reaches the chemisorbed $\mathrm{H}$ and recombines to form $\mathrm{H}_{2}$ and $\eta$ the probability to eject the molecular hydrogen formed. The incident $\mathrm{H}$ atom from the gas phase must hit the grain with enough thermal energy to cross the activation barrier $\left(E_{\mathrm{a}}\right)$ by thermal hopping. $f$ is given by:

$f=\frac{N_{\mathrm{c}}}{N} \times \exp \left(-\frac{E_{\mathrm{a}}}{k T_{\text {gas }}}\right)$

with $N_{\mathrm{c}}$ the total number of chemisorbed $\mathrm{H}$ atoms on the grain surface and $N$ the total number of physisorbed and chemisorbed sites. The fraction of chemisorbed $\mathrm{H}$ atoms $\left(N_{\mathrm{c}} / N\right)$ is critical for this process. This fraction will be significant when $T_{\text {gas }}$ is sufficiently high to carry an appreciable fraction of chemisorbed sites occupied. In PDRs (where $T_{\text {gas }} \geq$ $300 \mathrm{~K}$ ) we expect $N_{\mathrm{c}} / N$ to be relatively high while in cold interstellar clouds $N_{\mathrm{c}} / N$ should be low.

An alternative to the above is the case where the incident $\mathrm{H}$ atom initially sticks to a physisorbed site and then scans the surface until it "finds" the chemisorbed $\mathrm{H}$ atom. Here, the formation probability $P_{\mathrm{H}_{2}}$ can be written as:

$P_{\mathrm{H}_{2}}=S \times f \times \eta$

with $S$ the sticking probability.

The probability $f$ that the physisorbed $\mathrm{H}$ atom diffuses over the grain surface (until it reaches a neighbouring site to a chemisorbed $\mathrm{H}$ atom) and recombines before it is evaporated, can be given by $f=\frac{\tau_{\mathrm{p}}^{-1}}{\tau_{\mathrm{p}}^{-1}+\tau_{\mathrm{ev}}^{-1}}$ where $\tau_{\mathrm{p}}^{-1}$ is the inverse time scale for diffusing and recombining and $\tau_{\mathrm{ev}}^{-1}$ the evaporation rate of physisorbed $\mathrm{H}$. In the appendix we estimate the time scales $\tau_{\mathrm{p}}$ and $\tau_{\mathrm{ev}}$. This leads to the expression:

$$
f=\frac{1}{1+\left(\frac{N}{4 N_{\mathrm{c}}}\right)^{2} \times \exp \left(\frac{E_{\mathrm{p}}-E_{\mathrm{d}}}{k T_{\text {dust }}}\right)+\left(\frac{N}{4 N_{\mathrm{c}}}\right) \times k_{\mathrm{rec}}}
$$

with $E_{\mathrm{d}}$ the desorption energy of a physisorbed $\mathrm{H}$ atom and $E_{\mathrm{p}}$ the activation barrier energy for the diffusion of a physisorbed $\mathrm{H}$ (see Table 2). Considering that the physisorbed $\mathrm{H}$ atom crosses the activation barrier to recombine with a neighbouring chemisorbed atom by either (i) thermal diffusion or by (ii) tunnelling, we find (see appendix) that $k_{\text {rec }}=\exp \left(\frac{E_{\mathrm{a}}-E_{\mathrm{d}}}{k T_{\text {dust }}}\right)$ at high dust temperatures (above $\sim 40 \mathrm{~K}$ ) when thermal diffusion dominates whereas at low temperatures (below $\sim 30 \mathrm{~K}$ ) when tunneling is more important $k_{\text {rec }}=$ $\exp \left(E_{\mathrm{d}}\left(\frac{1}{k T_{\text {cr }}}-\frac{1}{k T_{\text {dust }}}\right)\right)$ with $k T_{\text {cr }}=80 E_{\mathrm{d} 3} /\left(E_{\mathrm{a} 3}^{0.5} \times \Delta x(\AA)\right)$ where $E_{\mathrm{a} 3}=E_{\mathrm{a}} /(1000 \mathrm{~K}), E_{\mathrm{d} 3}=E_{\mathrm{d}} /(1000 \mathrm{~K})$ and $\Delta x$ is the width of the barrier typically of the order of $\sim 1-3 \AA$ (Buch 1989; Fromherz et al. 1993; Sidis et al. 2000).

The activation barrier energies for physisorbed $\mathrm{H}$ atom diffusion $\left(E_{\mathrm{p}}\right)$ and for recombination with a neighbouring chemisorbed atom $\left(E_{\mathrm{a}}\right)$ are critical for this process. For $E_{\mathrm{a}}$ high $(\gtrsim 1000 \mathrm{~K})$, the recombination term will dominate and $f$ will be high only where tunneling dominates (see Fig. 5). For $E_{\mathrm{a}}$ low $(\sim 600 \mathrm{~K})$, the diffusion term will be critical and $f$ will depend much on $E_{\mathrm{p}}$. In this case, $f$ will be high for $E_{\mathrm{p}} \lesssim E_{\mathrm{d}}$ (if $E_{\mathrm{p}}$ is too large the atoms evaporate before they find a neighbouring chemisorbed site) and for $E_{\mathrm{p}} \sim 500 \mathrm{~K}$ we find that the fraction of chemisorbed $\mathrm{H}\left(N_{\mathrm{c}} / N\right)$ needs to be at least about 0.1 (see Sect. 6).

We note that formation by chemisorption can happen on the surface of big or small grains. In fact, the thermal fluctuations undergone by SGs after absorbing a UV photon are not sufficient to evaporate chemisorbed $\mathrm{H}$ atoms (binding energy around $10000 \mathrm{~K}$ ). Moreover, we estimate that the timescale for a physisorbed $\mathrm{H}$ to find a chemisorbed $\mathrm{H}$ is much less than the timescale of thermal fluctuations (see Sect. 5.1).

Since SGs make a dominant contribution to the total grain surface (see Sect. 6) and also because they may have numerous chemically bonded hydrogen atoms (because of their small 
Table 2. Parameters for carbonaceous dust surface.

\begin{tabular}{lll}
\hline \hline Parameter & Value & Ref. \\
\hline$E_{\mathrm{d}}(\mathrm{K})$ & 600 & $(1)$ \\
$E_{\mathrm{p}}(\mathrm{K})$ & 500 & $(1)$ \\
$E_{\mathrm{a}}(\mathrm{K})$ & $600-1000$ & $(2)-(3,4)$ \\
$\Delta x(\AA)$ & $\sim 2$ & $(2)$ \\
\hline
\end{tabular}

$E_{\mathrm{d}}$ is the desorption energy of a physisorbed $\mathrm{H}$ atom; $E_{\mathrm{p}}$ is the activation barrier energy for physisorbed $\mathrm{H}$ diffusion; $E_{\mathrm{a}}$ and $\Delta x$ are respectively the energy and the width of the activation barrier for recombination with a neighbouring chemisorbed $\mathrm{H}$. Here, we assume that the barrier $E_{\mathrm{a}}$ for recombination to form $\mathrm{H}_{2}$ is the same between a physisorbed and a chemisorbed site as for direct recombination from the gas phase.

References. (1) Katz et al. (1999); (2) Cazaux \& Tielens (2003); (3) Fromherz et al. (1993); (4) Parneix \& Brechignac (1998).

size they are more disordered), it is plausible that they play a dominant role in $\mathrm{H}_{2}$ formation by chemisorption. However, if small grains contribute efficiently to $\mathrm{H}_{2}$ formation, they must be continuously rehydrogenated : this could be the case for larger PAHs and VSGs for which the adsorbed $\mathrm{H}$ atoms should evaporate less after UV photon absorption because of the lower temperature fluctuations (see, e.g., Verstraete et al. 2001).

In the next section, we compare the $\mathrm{H}_{2}$ formation rates as predicted by the different mechanisms discussed here and as derived from observations.

\section{Comparison between the $\mathrm{H}_{2}$ formation rate as predicted and as derived from observations}

In this section, we see how the observational values of $\mathrm{H}_{2}$ formation rate in PDRs fit and constrain $\mathrm{H}_{2}$ formation mechanisms on grain surfaces.

First, based on the empirical formation probabilities given above, we estimate the rate of formation through chemisorption on small and big carbonaceous grains. The formation rate $R_{\mathrm{f}}$ (in $\mathrm{cm}^{3} \mathrm{~s}^{-1}$ ) can be written as:

$R_{\mathrm{f}}=\frac{1}{2} \sigma v_{\mathrm{H}} \times P_{\mathrm{H}_{2}}$

where $\sigma v_{\mathrm{H}}$ is the collision frequency between $\mathrm{H}$ atoms (with mean velocity $v_{\mathrm{H}}$ ) and grains and $P_{\mathrm{H}_{2}}$ the probability of formation (see Eqs. (3) and (5)). The mean cross section for collisions between grains and $\mathrm{H}$ atoms, $\sigma\left(\mathrm{cm}^{2}\right)$, is given by $<\frac{n_{\text {grain }}(a)}{n_{\mathrm{H}}} \pi a^{2}>$ with $n_{\text {grain }}(a)$ the grain density with radius between $a$ and $a+\mathrm{d} a$. We assume a MRN size distribution with $\alpha=3.5$ while the lower and upper limits of the grain radius are respectively $a_{\min }=4$ and $a_{\max }=100 \AA$ for SGs and $a_{\min }=$ 0.01 and $a_{\max }=0.1 \mu \mathrm{m}$ for BGs. Adopting uniform mass density $\rho=2.25 \mathrm{~g} \mathrm{~cm}^{-3}$ (typical value for graphite grains) and an grain/gas mass ratio $G$ about $0.001^{2}$ for SGs and 0.01 for BGs,

\footnotetext{
2 The carbon locked up in SGs has an abundance of $[\mathrm{C} / \mathrm{H}] \sim 10^{-4}$ inferred from comparison between observations of dust galactic emission and extinction with model calculations (Désert et al. 1990; Li \& Draine 2001).
}

we estimate ${ }^{3}$ : (i) for SGs, $\sigma v_{\mathrm{H}} \sim 6 \times 10^{-16}\left(\frac{T_{\mathrm{gas}}}{100 \mathrm{~K}}\right)^{0.5} \mathrm{~cm}^{3} \mathrm{~s}^{-1}$; (ii) for BGs, $\sigma v_{\mathrm{H}} \sim 3 \times 10^{-16}\left(\frac{T_{\text {gas }}}{100 \mathrm{~K}}\right)^{0.5} \mathrm{~cm}^{3} \mathrm{~s}^{-1}$. Under these assumptions, we find that the collisions cross section is $\sim 2$ higher for SGs than for BGs and that there is enough grain area with $\mathrm{SGs}$ to make $R_{\mathrm{f}}$ larger than the standard $\mathrm{H}_{2}$ formation rate. We assume the small dust abundance to be constant from one region to another although variations are expected. However, this assumption should not be critical for our approach which attempts with simple empirical models to see what type of $\mathrm{H}_{2}$ formation processes are to a first order relevant in PDRs.

To calculate $P_{\mathrm{H}_{2}}$, we adopt the following assumptions. Firstly, for the indirect chemisorption, the formation efficiency will depend on the sticking coefficient $S$ which remains uncertain. Studies of the sticking of $\mathrm{H}$ on grain surfaces generally predicts that $S$ is about 1 at low temperatures and decreases with increasing temperature to about $0.4-0.1$ at $T_{\text {gas }} \sim$ 300 K (Hollenbach \& McKee 1979; Burke \& Hollenbach 1983; Leitch-Devlin \& Williams 1985; Buch \& Zhang 1991). Here, we approximate $S$ by $1 /\left(1+T_{\text {gas }} / 400 \mathrm{~K}+\left(T_{\text {gas }} / 400 \mathrm{~K}\right)^{2}\right)$ from Burke \& Hollenbach (1983) and Bertoldi (1997). Secondly, for the parameters of the physisorbed and chemisorbed sites, we assume typical values for carbonaceous grains which are summarized in Table 2. We treat the surface coverage of chemisorbed $\mathrm{H}$ atoms $\left(N_{\mathrm{c}} / N\right)$ as a free parameter. Further, we take $N_{\mathrm{c}} / N$ for SGs and BGs to be equal and constant although this fraction is likely to depend upon grain characteristics and physical conditions. This is not completely satisfactory but it gives some insight into this parameter which more detailed theories of these processes should attempt to fit. We find that $N_{\mathrm{c}} / N \sim 0.1$ (see below) gives a reasonable fit to our PDR data. Finally, we take the probability to eject $\mathrm{H}_{2}, \eta$, to be unity. This is based simply on the fact that ejecting the newly formed $\mathrm{H}_{2}$ molecule (the desorption energy is $\sim 0.05 \mathrm{eV}$, Katz et al. 1999; Cazaux \& Tielens 2003) requires a small fraction of the available formation energy deposited in the grain $(1.5 \mathrm{eV})$. Note that if we do not assume spontaneous desorption, the $\mathrm{H}_{2}$ formed would stay on the surface for really low temperatures (below $10 \mathrm{~K}$ ) since the temperature is not high enough to allow evaporation (see Cazaux \& Tielens 2002, 2003).

For the purpose of comparison with the formation rates determined using PDR models (Sect. 3), we need to estimate dust temperatures in the PDRs of interest to us. In Table 3, we give the temperatures of SGs and BGs expected in the $\mathrm{H}^{0} / \mathrm{H}_{2}$ transition zone, where the radiation field is given by $\chi \exp (-\tau)$ with $\tau$ the FUV dust opacity in the transition zone equal to about unity. For the big grains at thermal equilibrium, we have adopted the analytic expression of Hollenbach et al. (1991). For the small grains subject to thermal fluctuations, we list in Table 3 the temperatures for a graphite grain of radius $1.5 \mathrm{~nm}(N(\mathrm{C}) \simeq 1600)$. We have computed for each value of the radiation field the full temperature histogram and the values in Table 3 correspond to the median value of the time

\footnotetext{
${ }^{3}$ We calculate $\sigma v_{\mathrm{H}}$ with $v_{\mathrm{H}}=\left(\frac{8 k T_{\mathrm{gas}}}{\pi m_{\mathrm{H}}}\right)^{1 / 2}$ and $\sigma=\int_{a_{\min }}^{a_{\max }} \pi a^{2} a^{-\alpha} \mathrm{d} a \times$ $\frac{V_{\text {grain } / \text { gas }}}{\max \frac{4}{3} \pi a^{3} a^{-\alpha} \mathrm{d} a}$ with $V_{\text {grain } / \text { gas }}=1.4 m_{\mathrm{H}} G / \rho$ the volume of grain per hydrogen nuclei.
} 
(a)

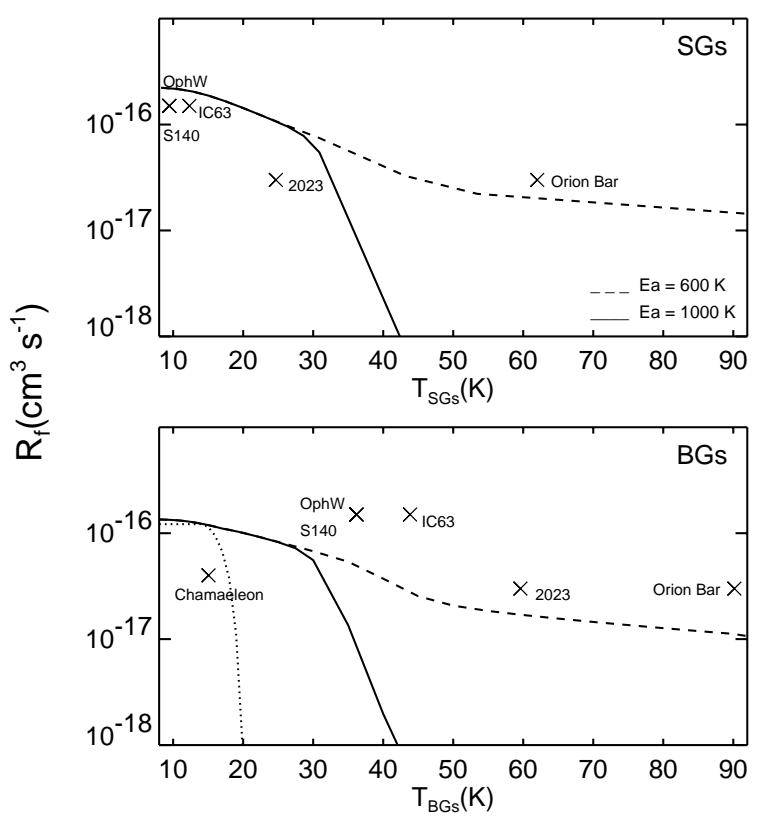

(b)
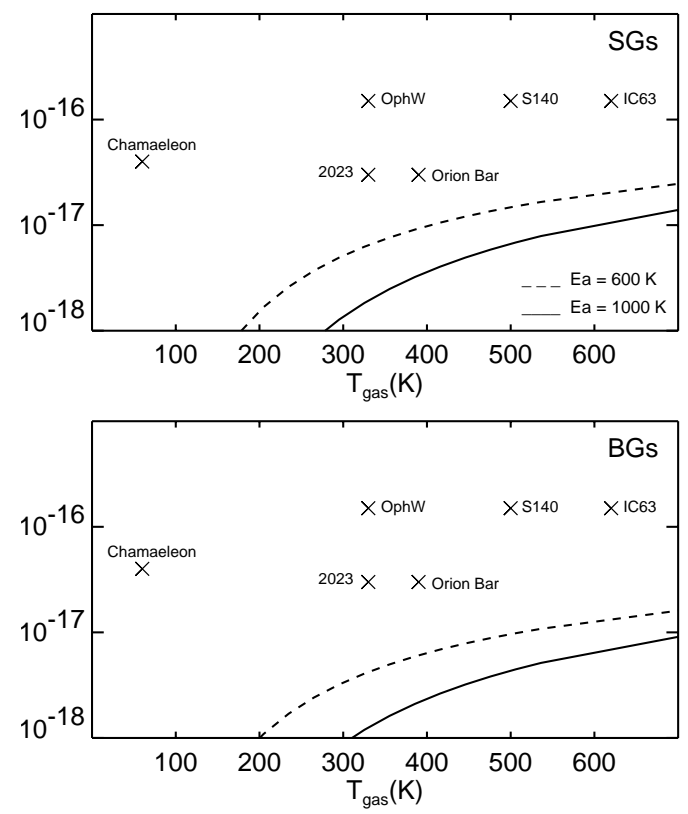

Fig. 5. Panel a): $\mathrm{H}_{2}$ formation rate as predicted for the indirect chemisorption mechanism (solid and dashed lines) and for the pure Langmuir-Hinshelwood mechanism (dotted lines) and as observed (crosses, see Table 3) as a function of the dust temperature. The dust temperatures for Oph W and S140 derived are similar (see Table 3). The upper and lower panels show the rates predicted respectively for formation on small and big grains. The solid and dashed lines correspond respectively to $E_{\mathrm{a}}$ equal to 1000 and $600 \mathrm{~K}$. Panel b): $\mathrm{H}_{2}$ formation rate as predicted for the $\mathrm{H}_{2}$ formation by direct chemisorption (solid and dashed lines) and as observed (crosses) as a function of the gas temperature. The "observed" gas temperatures are inferred from the distribution of low $\mathrm{H}_{2}$ pure rotational levels reported in Table 1; for the Chamaeleon see Gry et al. (2002).

Table 3. Temperatures of dust and gas and $\mathrm{H}_{2}$ formation rates.

\begin{tabular}{lllll}
\hline \hline Region & $\begin{array}{l}T_{\mathrm{SGs}}^{a} \\
(\mathrm{~K})\end{array}$ & $\begin{array}{l}T_{\mathrm{BGs}}^{a} \\
(\mathrm{~K})\end{array}$ & $\begin{array}{l}T_{\text {gas }}^{b} \\
(\mathrm{~K})\end{array}$ & $\begin{array}{l}R_{\mathrm{f}}{ }^{c} \\
\left(\mathrm{~cm}^{3} \mathrm{~s}^{-1}\right)\end{array}$ \\
\hline Chamaeleon & $>2.7$ & 15 & 60 & $4 \times 10^{-17}$ \\
& & & & \\
Oph W & 10 & 36 & 330 & $1.5 \times 10^{-16}$ \\
S140 & 10 & 36 & 500 & $1.5 \times 10^{-16}$ \\
IC 63 & 12 & 44 & 620 & $1.5 \times 10^{-16}$ \\
NGC 2023 & 25 & 60 & 330 & $3 \times 10^{-17}$ \\
Orion Bar & 62 & 90 & 390 & $3 \times 10^{-17}$ \\
\hline
\end{tabular}

${ }^{a}$ Temperature of the small grains (SGs) and big grains (BGs) expected in the $\mathrm{H}^{0} / \mathrm{H}_{2}$ transition zone (see text in Sect. 6).

${ }^{b}$ Gas temperature inferred from the distribution of low $\mathrm{H}_{2}$ pure rotational levels (see Table 1). For the Chamaeleon see Gry et al. (2002).

${ }^{c} \mathrm{H}_{2}$ formation rates derived from observations (see Sect. 3).

dependent temperature (i.e., the grain spends half of the time at temperature higher and lower than this value). The median temperature of small grains is unlike that of big grains not constrained by their emission spectrum. In these calculations, we used the absorption and emission cross section of bulk graphite from Draine \& Lee (1984); the heat capacity is also of graphite given by Guhathakurta \& Draine (1989). We have thus ignored quantum effects which might significantly change properties of these particles. This estimate of the small grain temperature is only indicative. Ideally, one should take into account the time dependence of the temperature as a function of the grain size.
Further, their emission properties might not allow then to cool down to the cosmic $2.7 \mathrm{~K}$ background temperature. Moreover, for very low temperatures $(\leq 10 \mathrm{~K})$, the heating due to the collisions between the grains and warm gas ( $\left.T_{\text {gas }} \geq 100 \mathrm{~K}\right)$ or due to the formation of $\mathrm{H}_{2}(\sim 1 / 3$ of the formation energy is deposited in the grain) would increase the grain temperature. However, in the following we will use the SG temperatures reported in Table 3 in order to see if small grains are relevant for forming $\mathrm{H}_{2}$. We find that the median temperatures of small grains are significantly lower than those of big grains. Indeed, SGs spend most of their time at low temperature (Guhathakurta \& Draine 1989).

With the above formalism, we compute the formation rate $R_{\mathrm{f}}$ for the various processes outlined above on both SGs and BGs. The results are presented in Fig. 5, where we compare the $\mathrm{H}_{2}$ formation rate predicted for indirect and direct chemisorption with the rates derived from observations. For completeness, we also show the results for the case of the pure Langmuir-Hinshelwood mechanism upon BGs using the model of Katz et al. (1999) based on experimental results for graphite surfaces (Pirronello et al. 1999).

First, we see from Fig. 5a that indirect chemisorption may explain the data for all sources if the activation barrier energy for recombination is $E_{\mathrm{a}} \lesssim 1000 \mathrm{~K}$ and if the fraction of chemisorbed $\mathrm{H}$ atoms is as large we have assumed, $N_{\mathrm{c}} / N \sim$ 0.1 . This result is broadly consistent with the recent theoretical model of Cazaux \& Tielens (2002, 2003), which accounts properly for the population of chemisorbed and physisorbed sites on 
the surface. Thus, our estimate of the fraction of chemisorbed $\mathrm{H}$ atoms may be reasonable. However, the value of the activation barrier energy is uncertain and critically influences our estimate for $R_{\mathrm{f}}$ and the temperature range for which formation via indirect chemisorption is important. We find in fact that for $E_{\text {a }} \sim 1000 \mathrm{~K}$ this process will be efficient until $T_{\text {dust }} \sim 30 \mathrm{~K}$ (i.e., important in PDRs of relatively moderate excitation, $\chi<$ 5000); for $T_{\text {dust }}>30 \mathrm{~K}$ the physisorbed $\mathrm{H}$ atoms evaporate before recombining. Conversely, for $E_{\mathrm{a}} \sim 600 \mathrm{~K}$ the formation via indirect chemisorption will be still efficient at $T_{\text {dust }} \sim 100 \mathrm{~K}$ (i.e., important even in highly excited PDRs). In this last case, where the diffusion term dominates (see Sect. 5.2), the decreasing of $R_{\mathrm{f}}$ from moderate to highly excited PDRs is due to the competition between evaporation and finding the chemisorbed $\mathrm{H}$ atoms over the surface.

Secondly, we find that small grains which dominate the total grain area and spend most of their time at low temperature $(<30 \mathrm{~K}$ for $\chi \leq 3000$, see Table 3$)$ may be the most promising surface for forming $\mathrm{H}_{2}$ via indirect chemisorption. The $\mathrm{H}_{2}$ formation on SGs can be effective even in highly excited PDRs where the equilibrium temperature of BGs can be too high to form $\mathrm{H}_{2}$. In the case of SGs, we note that in most objects, the observed $\mathrm{H}_{2}$ formation rates can be explained with a high value of $E_{\mathrm{a}}(\sim 1000 \mathrm{~K})$. For BGs, conversely a low value of $E_{\mathrm{a}}(\$ 600 \mathrm{~K})$ is required. However, our description of the temperature of SGs is crude (median value of the temperature distribution for a single size of grain). As a consequence, we cannot quantitatively constrain the parameters $\left(E_{\mathrm{a}}, E_{\mathrm{p}}, \ldots\right)$ of the $\mathrm{H}_{2}$ formation process.

The direct or Eley-Rideal mechanism fails to explain the observations by a factor of a few (see Fig. 5b) although a higher $N_{\mathrm{c}} / N$ combined with a slightly lower $E_{\mathrm{a}}$ would suffice to explain some of the data. We cannot therefore exclude this mechanism but find that our present data suggest that the indirect chemisorption on SGs is more probable.

There are several complications which we have neglected in the above treatment. One is the possible presence of interstitial sites as suggested by Duley (1996) which can increase the efficiency of direct chemisorption. Another perhaps is $\mathrm{H}_{2}$ formation through a reaction between two chemisorbed $\mathrm{H}$ atoms as suggested by Cazaux \& Tielens $(2002,2003)$. This mechanism would be efficient at high dust temperatures $(\gtrsim 100 \mathrm{~K})$ and could be a possibility to explain the formation of $\mathrm{H}_{2}$ in Orion.

In summary, we conclude that in order to explain the $\mathrm{H}_{2}$ formation efficiency in PDRs, the indirect chemisorption mechanism upon small grains is the most promising. This requires an activation barrier energy between a physisorbed $\mathrm{H}$ atom and a neighbouring chemisorbed $\mathrm{H}$ atom $E_{\mathrm{a}} \lesssim 1000 \mathrm{~K}$ (or $\lesssim 0.1 \mathrm{eV}$ ) and a fraction of occupied chemisorbed sites of around ten percent. This conclusion is consistent with our finding of a correlation between the $\mathrm{H}_{2}$ and $\mathrm{PAH}$ emission which suggests that $R_{\mathrm{f}}$ scales with the PAH abundance (see Sect. 4). However, a better knowledge of the SGs properties (temperature, coverage of absorbed $\mathrm{H}$ atom, abundance ...) and of the mobilities of $\mathrm{H}$ atoms on realistic grain surfaces are critical.

\section{Conclusion}

The main aim of this study has been to provide estimates of the molecular hydrogen formation rate in a sample of nearby PDRs using results from both ISO and ground-based telescopes. The physical conditions in the PDR layers from which $\mathrm{H}_{2}$ emission is observed $\left(n_{\mathrm{H}} \simeq 10^{3}-10^{5} \mathrm{~cm}^{-3}, T_{\text {gas }} \geq 300 \mathrm{~K}\right.$ ) differ considerably from those in the diffuse clouds where one can observe the UV lines of $\mathrm{H}_{2}\left(n_{\mathrm{H}} \simeq 100-1000 \mathrm{~cm}^{-3}, T_{\text {gas }} \simeq 50-100 \mathrm{~K}\right)$ and upon which most estimates of the molecular hydrogen formation rate have been based. Thus the results from PDRs allow important constraints to be placed upon the mechanisms for forming molecular hydrogen on grain surfaces. We confirm the earlier result of Habart et al. (2003) that the $\mathrm{H}_{2}$ formation rate in regions of moderate excitation $(\chi \leq 1000)$ such as Oph W, S140 and IC 63 is a factor of $\sim 5$ times larger than the standard rate estimated by Jura for diffuse clouds (and confirmed by recent FUSE data). On the other hand, towards regions of higher radiation field such as the Orion Bar and NGC 2023, we derive $\mathrm{H}_{2}$ formation rates consistent with the standard value. Thus, the higher grain and gas temperatures in PDRs do not seem to impede the formation of $\mathrm{H}_{2}$.

We have attempted to interpret these results with simple empirical models of the formation of $\mathrm{H}_{2}$ on grain surfaces. From these, we conclude that an "indirect chemisorption" model analogous to that discussed by Cazaux \& Tielens (2002, 2003) is capable of explaining the data. This result requires an activation barrier energy against the recombination of a physisorbed $\mathrm{H}$ atom and a neighbouring chemisorbed $\mathrm{H}$ atom $E_{\mathrm{a}} \lesssim 0.1 \mathrm{eV}$. Another condition appears to be that one needs an appreciable fraction of surface sites occupied (few percent at least) with a binding energy of order $1 \mathrm{eV}$ relative to the total number of surface binding sites (presumably mainly physisorbed with binding energies of order $0.05 \mathrm{eV}$ ). Moreover, we suggest that small (size $<100 \AA$ ) grains may be the most promising surface for forming $\mathrm{H}_{2}$ in PDRs. There is in fact enough grain surface in small grains to allow the formation rate to be larger than the standard $\mathrm{H}_{2}$ formation rate and small grains spend most of their time at low temperature (Guhathakurta \& Draine 1989). $\mathrm{H}_{2}$ formation by indirect chemisorption upon small grains should be effective even in highly excited regions where large grains are quite warm.

Our results show that formation of molecular hydrogen in PDRs is likely to take place with a different mechanism than in the diffuse interstellar medium where the formation by physisorbed $\mathrm{H}$ atoms (Langmuir-Hinshelwood) probably dominates. In fact, in cold diffuse clouds the surface density of strongly bound $\mathrm{H}$ atoms should be low and consequently the formation by chemisorption would not be efficient.

There are several fundamental uncertainties in our present estimates of the $\mathrm{H}_{2}$ formation rates in PDRs which future work should try to eliminate. One is due to the fact that we have used steady-state PDR models. This assumption can cause appreciable errors and it would be useful to calculate the expected $\mathrm{H}_{2}$ line intensities for models with lower $\mathrm{H}_{2}$ formation rates but where advection has been taken into account. However, Störzer \& Hollenbach (1998) have modelled PDR structure assuming an ionization front moving into the PDR and found that 
non-equilibrium effects are probably minor in objects similar to the Orion Bar.

Other uncertainties are of an observational nature. It would be useful to have reliable estimates of the inclination and density distribution in the PDRs of interest in order to better constrain the models. There are indications in the Orion Bar for instance (Walmsley et al. 2000) that the column density is higher in the molecular layers of the PDR than in the region (discussed in this paper) where the $\mathrm{H}_{2}$ lines are formed. Density gradients perpendicular to the PDR photodissociation front clearly need to be taken into account when considering the spatial distribution of the various $\mathrm{H}_{2}$ lines.

The theoretical models of $\mathrm{H}_{2}$ formation discussed here are clearly very preliminary. More detailed models need to explicitly consider the degree of occupation of chemisorbed sites by $\mathrm{H}$-atoms as well as the mobility of $\mathrm{H}$ atoms on various types of grain surfaces. Our results however do suggest that more detailed consideration of $\mathrm{H}_{2}$ formation on the surface of small grains would be worthwhile. In this case, one should ideally follow the thermal fluctuations of these small particles and take into account the dependence on grain size in order to examine properly their contribution to $\mathrm{H}_{2}$ formation. Clearly also our present estimates for processes such as tunneling are very crude.

Additional information on the $\mathrm{H}_{2}$ formation process could probably be obtained using high quality data for $\mathrm{H}_{2}$ in excited vibrational states which gives constraints on the excitation state of the newly formed $\mathrm{H}_{2}$. It also would be useful to obtain estimates of the $\mathrm{H}_{2}$ formation rate under a variety of different conditions. One such condition might be in the Magellanic clouds where the different metallicity, extinction curve, and radiation field potentially may influence both the available grain surface area and the efficiency of $\mathrm{H}_{2}$ formation. Another is in the thin high excitation clouds such as that found by Meyer et al. (2001) towards the exciting star of NGC 2023. It is clear that given our uncertainty about grain compositions and size distributions in different ISM locations, one is forced to some extent to use the astrophysical data to guide our estimates of processes such as $\mathrm{H}_{2}$ formation. This is perhaps philosophically not as satisfactory as the traditional approach of employing experimentally determined or theoretically calculated rates but it is likely that nature does not give us the choice.

Acknowledgements. We are grateful to the referee, David Hollenbach, for relevant comments and suggestions. We also thank Stéphanie Cazaux and Eric Herbst for fruitful discussion on the $\mathrm{H}_{2}$ formation process. C.M.W. wish to acknowledge travel support from the MUIR project "Dust and Molecules in Astrophysics Environments".

\section{Appendix: Probability for the diffusion and recombination of a physisorbed $\mathrm{H}$ atom with a chemisorbed $\mathrm{H}$ atom}

To calculate the probability $f=\frac{\tau_{\mathrm{p}}^{-1}}{\tau_{\mathrm{p}}^{-1}+\tau_{\mathrm{ev}}^{-1}}$ (Eq. (6), see Sect. 5.2), we estimate the timescale $\tau_{\mathrm{p}}$ for diffusion over the grain surface followed by recombination and the timescale $\tau_{\mathrm{ev}}$ for evaporation of the physisorbed $\mathrm{H}$. The latter time scale is given by

$$
\tau_{\mathrm{ev}}=v_{0}^{-1} \exp \left(\frac{E_{\mathrm{d}}}{k T_{\text {dust }}}\right)
$$

with $E_{\mathrm{d}}$ the desorption energy of a physisorbed $\mathrm{H}$ and $v_{0}$ the vibrational frequency of $\mathrm{H}$ in a physisorbed site typically of the order of $10^{12} \mathrm{~s}^{-1} \cdot \tau_{\mathrm{p}}$ can be given by the sum of the time to find a neighboring site to a chemisorption site bearing a $\mathrm{H}$ atom, $\tau_{\mathrm{m}}$ and of the time to recombine and form $\mathrm{H}_{2}, \tau_{\text {rec }}$. We assume that diffusion from one physisorbed site to another occurs by thermal hopping (Katz et al. 1999). The time to hop from one physisorbed site to the next is thus given by $v_{0}^{-1} \exp \left(\frac{E_{\mathrm{p}}}{k T_{\text {dust }}}\right)$ with $E_{\mathrm{p}}$ the activation barrier energy for physisorbed $\mathrm{H}$ atom diffusion. The mobility is a random walk and considering that there are 4 neighboring sites for each chemisorbed site, we find that one needs approximately $\left(N / 4 N_{\mathrm{c}}\right)^{2}$ steps to be adjacent to a filled chemisorbed site. Thus:

$\tau_{\mathrm{m}}=\left(\frac{N}{4 N_{\mathrm{c}}}\right)^{2} \times v_{0}^{-1} \exp \left(\frac{E_{\mathrm{p}}}{k T_{\text {dust }}}\right)$.

The physisorbed $\mathrm{H}$ atom must cross the activation barrier to recombine with the nearest chemisorbed $\mathrm{H}$ atom by either (i) thermal diffusion with a probability $f_{\text {th }}=\exp \left(-\frac{E_{\mathrm{a}}}{k T_{\text {dust }}}\right)$ or by (ii) tunnelling with a probability $f_{\text {tun }}=\exp \left(-\frac{2 \Delta x}{\hbar}\left(2 m_{\mathrm{H}} E_{\mathrm{a}}\right)^{0.5}\right)$ (Messiah 1972) with $\Delta x$ the width of the barrier. The recombination time scale can be approximated by

$\tau_{\text {rec }}=\left(\frac{N}{4 N_{\mathrm{c}}}\right) \times v_{0}^{-1} \frac{1}{\left(f_{\text {th }}+f_{\text {tun }}\right)}$.

At high dust temperatures (above $\sim 40 \mathrm{~K}$ ) thermal diffusion dominates whereas at low temperature (below $\sim 30 \mathrm{~K}$ ) tunneling is more important.

There are many caveats to the above procedure. In particular, we note that considering the probability $\exp \left(-\frac{2 \Delta x}{\hbar}\left(2 m_{\mathrm{H}} E_{\mathrm{a}}\right)^{0.5}\right)$ for tunneling transmission through a single barrier is incorrect for relevant astrophysical surfaces which are not regular and need therefore to be modeled by a set of barriers as many as there are atoms. The tunneling probability we adopt is thus an upper limit. However, for the purpose of comparison with observationally determined rates, our approach allows us to see which of the $\mathrm{H}_{2}$ formation processes considered in this study will dominate.

\section{References}

Abergel, A., Bernard, J. P., Boulanger, F., et al. 2002, A\&A, 389, 239

Bakes, E. L. O., \& Tielens, A. G. G. M. 1994, ApJ, 427, 822

Bertoldi, F. 1997, in First ISO Workshop on Analytical Spectroscopy, 67

Biham, O., Furman, I., Pirronello, V., \& Vidali, G. 2001, ApJ, 553, 595

Black, J. H., \& van Dishoeck, E. F. 1987, ApJ, 322, 412

Boulanger, F., Boisssel, P., Cesarsky, D., \& Ryter, C. 1998, A\&A, 339, 194

Boulanger, F., Rubio, M., Habart, E., \& Verstraete, L. 2003, in preparation

Buch, V., \& Zhang, Q. 1991, ApJ, 379, 647 
Buch, V. 1989, Proc. Int. School of Physics "Enrico Fermi", course CI, 321, ed. A. Bonetti, \& J. M. Greenberg (Elsevier Science Pub.) Burke, J. R., \& Hollenbach, D. J. 1983, ApJ, 265, 223

Burton, M. G., Howe, J. E., Geballe, T. R., \& Brand, P. W. J. L. 1998, PASAM, 15, 194

Cazaux, S., \& Tielens, A. G. G. M. 2002, ApJ, 575, L29

Cazaux, S., \& Tielens, A. G. G. M. 2003, ApJ, submitted

Cesarsky, D., Jones, A. P., Lequeux, J., \& Verstraete, L. 2000, A\&A, 358,708

Combes, F., \& Pineau des Forêts, G. 2002, Molecular Hydrogen in Space (Cambridge University Press)

Désert, F. X., Boulanger, F., \& Puget, J. L. 1990, A\&A, 237, 215

Draine, B. T., \& Bertoldi, F. 1996, ApJ, 468, 269

Draine, B. T., \& Bertoldi, F. 1999, in The Universe as seen by ISO, 553

Draine, B., \& Bertoldi, F. 2000, in Molecular Hydrogen in Space, 131

Draine, B. T., \& Lee, H. M. 1984, ApJ, 285, 89

Draine, B. T. 1978, ApJS, 36, 595

Draine, B. T. 1989, in Infrared Spectroscopy in Astronomy, 93

Duley, W. W., \& Williams, D. A. 1984, in Interstellar Chemistry

Duley, W. W. 1996, MNRAS, 279, 591

Field, D., Lemaire, J. L., Pineau Des Forêts, G., et al. 1998, A\&A, 333,280

Fromherz, T., Mendoza, C., \& Ruette, F. 1993, MNRAS, 263, 851

Fuente, A., Martin-Pintado, J., \& Rodriguez-Fernandez, N. 1999, ApJ, 518, L45

Gould, R. J., \& Salpeter, E. E. 1963, ApJ, 138, 393

Gry, C., Boulanger, F., Nehmé, C., et al. 2002, A\&A, 391, 675

Guhathakurta, P., \& Draine, B. T. 1989, ApJ, 345, 230

Habart, E., Verstraete, L., Boulanger, F., et al. 2001, A\&A, 373, 702

Habart, E., Boulanger, F., Verstraete, L., et al. 2003, A\&A, 397, 623

Habart, E. 2001, Ph.D Thesis, Couplage entre le gaz et les grains dans le milieu interstellaire, University of Paris 7

Herrmann, F., Madden, S. C., Nikola, T., et al. 1997, ApJ, 481, 343

Hogerheijde, M. R., Jansen, D. J., \& Van Dishoeck, E. F. 1995, A\&A, 294, 792

Hollenbach, D., \& McKee, C. F. 1979, ApJS, 41, 555

Hollenbach, D., \& Salpeter, E. E. 1971, ApJ, 163, 155

Hollenbach, D. J., \& Tielens, A. G. G. M. 1999, Rev. Mod. Phys., 71, 173

Hollenbach, D. J., Takahashi, T., \& Tielens, A. G. G. M. 1991, ApJ, 377, 192

Jansen, D. J., van Dishoeck, E. F., \& Black, J. H. 1994, A\&A, 282, 605

Jansen, D. J., van Dishoeck, E. F., Black, J. H., Spaans, M., \& Sosin, C. 1995, A\&A, 302, 223

Joblin, C., Boissel, P., Pech, C., Armengaud, M., \& Frabel, P. 2001, in Infrared and Submillimeter Space Astronomy (EDP Sciences)

Jura, M. 1975, ApJ, 197, 575

Katz, N., Furman, I., Biham, O., Pirronello, V., \& Vidali, G. 1999, ApJ, 522, 305

Kessler, M. F., Steinz, J. A., Anderegg, M. E., et al. 1996, A\&A, 315, L27

Le Bourlot, J., Pineau des Forêts, G., Roueff, E., \& Flower, D. R. 1993 , A\&A, 267, L233

Leitch-Devlin, M. A., \& Williams, D. A. 1985, MNRAS, 213, 295

Li, A., \& Draine, B. T. 2001, ApJ, 554, L778

Li, W., Evans, N. J. I, Jaffe, D. T, van Dishoeck, E. F., \& Thi, W. F. 2002, ApJ, 568, 242
Luhman, M. L., Luhman, K. L., Benedict, T., Jaffe, D. T., \& Fischer, J. 1997, ApJ, 480, L133

Marconi, A., Testi, L., Natta, A., \& Walmsley, C. M. 1998, A\&A, 330, 696

Martin, P. G., \& Mandy, M. E. 1995, ApJ, 455, L89

Messiah, A. 1972, Quantum mechanics (Amsterdam: North-Holland Publishing Company)

Meyer, D. M., Lauroesch, J. T., Sofia, U. J., Draine, B. T., \& Bertoldi, F. 2001, ApJ, 553, L59

Minchin, N. R., White, G. J., \& Padman, R. 1993, A\&A, 277, 595

Moos, H. W., Cash, W. C., Cowie, L. L., et al. 2000, ApJ, 538, L1

Motte, F., Andre, P., \& Neri, R. 1998, A\&A, 336, 150

Moutou, C., Verstraete, L., Sellgren, K., \& Léger, A. 1999, in The Universe as Seen by ISO, 727

Parneix, P., \& Brechignac, P. 1998, A\&A, 334, 363

Pirronello, V., Liu, C., Shen, L., \& Vidali, G. 1997, ApJ, 475, L69

Pirronello, V., Liu, C., Roser, J. E., \& Vidali, G. 1999, A\&A, 344, 681

Puget, J. L., Léger, A., \& Boulanger, F. 1985, A\&A, 142, L19

Rachford, B. L., Snow, T. P., Tumlinson, J., et al. 2001, ApJ, 555, 839

Rachford, B. L., Snow, T. P., Tumlinson, J., et al. 2002, ApJ, 577, 221

Sandford, S. A., \& Allamandola, L. J. 1993, ApJ, 409, L65

Sellgren, K., Allamandola, L. J., Bregman, J. D., Werner, M. W., \& Wooden, D. H. 1985, ApJ, 299, 416

Shull, J. M., Tumlinson, J., Jenkins, E. B., et al. 2000, ApJ, 538, L73

Sidis, V., Jeloica, L., Borisov, A., \& Deutscher, S. 2000, in Molecular Hydrogen in Space (Cambridge University Press)

Simon, R., Stutzki, J., Sternberg, A., \& Winnewisser, G. 1997, A\&A, 327, L9

Snow, T. P., Rachford, B. L., Tumlinson, J., et al. 2000, ApJ, 538, L65

Sonnentrucker, P., Friedman, S. D., Welty, D. E., York, D. G., \& Snow, T. P. 2002, ApJ, 576, 241

Störzer, H., \& Hollenbach, D. 1998, ApJ, 495, 853

Takahashi, J., Masuda, K., \& Nagaoka, M. 1999, ApJ, 520, 724

Tauber, J. A., Tielens, A. G. G. M, Meixner, M., \& Foldsmith, P. F. 1994, ApJ, 422, 136

Thi, W. F., van Dishoeck, E. F., Black, J. H., et al. 1999, in The Universe as Seen by ISO, 529

Tielens, A. G. G. M., \& Hollenbach, D. 1985, ApJ, 291, 747

Tielens, A. G. G. M., Meixner, M. M., van der Werf, P. P., et al. 1993, Science, 262, 86

Timmermann, R., Bertoldi, F., Wright, C. M., et al. 1996, A\&A, 315, L281

Tumlinson, J., Shull, J. M., Rachford, B. L., et al. 2002, ApJ, 566, 857

van der Werf, P. P., Stutzki, J., Sternberg, A., \& Krabbe, A. 1996, A\&A, 313, 633

Verstraete, L., \& Léger, A. 1992, A\&A, 266, 513

Verstraete, L., Pech, C., Moutou, C., et al. 2001, A\&A, 372, 981

Walmsley, C. M., Natta, A., Oliva, E., \& Testi, L. 2000, A\&A, 364, 301

Weingartner, J. C., \& Draine, B. T. 2001, ApJS, 134, 263

Williams, D., Clary, D. C., Farebrother, A., et al. 2000, in Molecular Hydrogen in Space, 13

Wyrowski, F., Schilke, P., Hofner, P., \& Walmsley, C. M. 1997a, ApJ, 487, L171

Wyrowski, F., Walmsley, C. M., Natta, A., \& Tielens, A. G. G. M. 1997b, A\&A, 324, 1135

Wyrowski, F., Walmsley, C. M., Goss, W. M., \& Tielens, A. G. G. M. 2000, ApJ, 543, 245 